\title{
L'histoire sous forme graphique, en arabe, persan, et turc ottoman
}

Origines et fonctions

\section{Denise Aigle}

\section{OpenEdition}

\section{Journals}

Édition électronique

URL : http://journals.openedition.org/beo/58

DOI : $10.4000 /$ beo.58

ISBN : 978-2-35159-316-5

ISSN : 2077-4079

\section{Éditeur}

Presses de l'Institut français du Proche-Orient

\section{Édition imprimée}

Date de publication : 1 septembre 2009

Pagination : 11-49

ISBN : 978-2-35159-143-7

ISSN : 0253-1623

\section{Référence électronique}

Denise Aigle, «L'histoire sous forme graphique, en arabe, persan, et turc ottoman », Bulletin d'études orientales [En ligne], Tome LVIII I Septembre 2009, mis en ligne le 01 septembre 2010, consulté le 19 avril 2019. URL : http://journals.openedition.org/beo/58 ; DOI : 10.4000/beo.58 


\author{
L'HISTOIRE SOUS FORME GRAPHIQUE \\ EN ARABE, PERSAN ET TURC OTTOMAN \\ Origines et fonctions *
}

Denise AIGLE

CNRS UMR 8167 «Orient et Méditerranée », EPHE-Sorbonne

La recherche présentée dans cet article est totalement originale parce que, à ma connaissance, personne n'a travaillé sur l'histoire rédigée sous une forme graphique combinant généalogies, textes narratifs et tableaux (taqwìm ou ğadwal). À vrai dire, il s'agit d'un domaine complexe. En effet, aucun texte de cette nature n'a été édité et, lorsque cela a été réalisé, rares sont les cas où les éditeurs ont respecté la présentation manuscrite. Il faut donc faire de longues recherches dans les catalogues de manuscrits pour tenter de dénicher des textes historiques présentés sous cette forme. Plusieurs questions se posent sur cette manière d'écrire l'histoire : à quel moment ces textes apparaissent-il dans l'historiographie islamique? Dans quelle aire géographique spécifique: historiographie arabe, persane, turque ? Pour quelles raisons? Pour répondre à la demande d'un public à un moment donné de l'histoire? Pour des raisons pédagogiques et/ou politiques? Enfin, peut-on déterminer l'origine (ou les origines) de ce mode d'écriture de l'histoire ? Donc, beaucoup de questions auxquelles je tenterai de proposer quelques éléments de réponse sous la forme d'hypothèses qui pourraient constituer des pistes pour des recherches ultérieures.

Dans un premier temps, je présenterai quelques textes non historiques qui utilisent des tableaux ou qui combinent texte et éléments graphiques. Très tôt, les taqwìm ont été adoptés pour établir des zĭğ, c'est-à-dire des manuels d'astronomie contenant des tables s'inspirant de différents modèles comme le Zīk-i Šahryār d'époque sassanide, le Sindhind indien ou encore l'Almageste et les Tables pratiques de Ptolémée. L'objectif de ces manuels était de fournir aux astronomes les données mathématiques qui leur étaient nécessaires pour calculer les positions (longitudes et latitudes) du soleil, de la lune et des cinq principales planètes ${ }^{1}$. Il semble cependant que, bien qu'héritier de la tradition ptoléméenne, l'origine

\footnotetext{
* Je remercie Michele Bernardini, Louise Marlow et Gilles Veinstein qui ont bien voulu relire une première version de cet article et l'ont enrichie de leurs remarques.

1. Sur les ziǧ dans l'islam, voir D. A. KING et J. SAMSÒ, « Zīdj », EI $I^{2}$, vol. XI, p. 537-550, où l'on trouvera une abondante bibliographie et des reproductions de manuscrits. Sur les ouvrages anciens de ce type, voir les travaux de D. PIN-
} 
des canons chronologiques établis par al-Bīrūnī ${ }^{2}$ (m. après 442/1050) dans son ouvrage al$\bar{A} t \bar{a} r$ al-bāqiyya ${ }^{3}$, remonte pour une partie à l'historien chrétien de langue grecque, Eusèbe de Césarée (m. ca. 332). En effet, al-Bīrūnī le cite de manière indirecte pour le calcul des ères rūmī. D'après Édouard C. Sachau, l'éditeur du texte, la mention d'Eusèbe de Césarée dérive du Ziğ de Yūsuf b. Faḍl al-Yahūdī al-Haybarī. On trouve antérieurement et postérieurement à al-Bīrūnī cette tradition dans l'historiographie syriaque qui est directement héritière des canons chronologiques d'Eusèbe de Césarée ${ }^{4}$ comme, par exemple, dans la Chronographie bilingue, en syriaque et en arabe, de l'historien nestorien ${ }^{5}$ Élie de Nisibie (m. 1046) ${ }^{6}$ qui le cite à plusieurs reprises et dans la chronique du patriarche d'Antioche, Michel le Syrien (m. 1199) ${ }^{7}$.

En ce qui concerne les textes historiques rédigés sous la forme de taqwìm qui combinent presque toujours histoire narrative et tableaux, il semble que ce soit à partir du XIII ${ }^{\mathrm{e}}-\mathrm{XIV}^{\mathrm{e}}$ siècle que ces ouvrages apparaissent plus systématiquement et, comme on pourra le constater, cette tradition est attestée jusqu'au XIX ${ }^{\mathrm{e}}$ siècle dans l'Empire ottoman. À partir du XII siècle, nous avons l'attestation de quelques textes historiques rédigés sous cette forme, ou qui, même s'ils ne nous sont pas parvenus, d'après le titre, semblent être des taqwìm. Je citerai l'exemple du Muğmal al-tawārīh wa l-qișaș, une histoire universelle qui, d'après le manuscrit conservé à la Bibliothèque nationale de France, fut composée en 520/1126 et dont l'auteur était le petit-fils d'un certain Muhallab b. Muhammad b. Šādī,

GREE, « Historical Horoscopes », Journal of the American Oriental Society, vol. 82 (1962), p. 487-502 ; D.A. KING, « On the Astronomical Tables of the Islamic Middle Ages ", in Colloquia Copernicana III, 1975, p. 37-56.

2. Sur cet important savant de l'Iran oriental, voir D. J. Borloт, « al-Bīrūnī », EI², vol. I, p. 1273-1275.

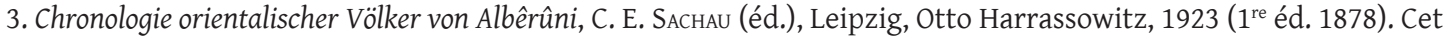
ouvrage ne traite pas seulement des calendriers et des ères, mais l'auteur aborde également des questions mathématiques.

4. Sur l'usage d'Eusèbe de Césarée par les chroniqueurs syriaques, voir M. DEBIÉ, « L'héritage de la chronique d'Eusèbe dans l'historiographie syriaque », Journal of the Canadian Society for Syriac Studies, vol. 6 (2006), p. 18-26.

5. Le terme "nestorien" est employé dans les sources médiévales, mais il a un sens péjoratif aux yeux de cette communauté qui ne s'est jamais désignée par ce nom : il s'agit de l'Église apostolique d'Orient.

6. Édition du texte, Eliae metropolitae Nisibeni. Opus chronologicum, E. W. ВRоокs et J.-B. СНавот (éd.), 2 vol., Paris, 19091910 (Corpus scriptorum christianorum orientalium, 62-63. Scriptores Syr. 21-24). Il en existe une traduction latine, voir La chronographie d'Élié Bar Šinaya. Métropolitain de Nisibe. Traduite pour la première fois d'après le manuscrit Add. 7197 du Musée britannique par L.-J. Delaporte, Paris, Honoré Champion, 1910.

7. Chronique de Michel le Syrien. Patriarche jacobite d'Antioche (1166-1199), 4 vol., J.-B. Снавот (éd. et trad. du fac-similé), Paris, Ernest Leroux, 1899-1910 [Réimpression anastatique de l'édition de Paris, Bruxelles, Culture et civilisation, 1963]. Sur cette chronique, voir D. WeLtecKE, Die «Beschreibung der Zeiten» von Mör Michael dem Grossen (1129-1199). Eine Studie zu ihrem historischen und historiographie geschichtlichen Kontext, Louvain, Peeters, 2003 (Corpus scriptorum christianorum orientalium, 594. Subsidia, t. 110). 
mais qui ne se nomme pas ${ }^{8}$. Outre l'histoire narrative, le texte comporte des tableaux ${ }^{9}$, des dessins géométriques, en marge, plusieurs cartes et une peinture ${ }^{10}$. On trouve, par exemple, au fol. 278v, un tableau intitulé « Tabaqat al-țālațat Banī 'Abbās » qui comporte huit rubriques, lues de droite à gauche ; elles portent ces intitulés : asmā', al-alqāb, asmāa, al-alqāa, $i b n-h \bar{a}$ et $a l q \bar{a} b^{11}$. Dans ces huit rubriques sont disposés dans les huit cases correspondantes les noms des personnages selon un mode graphique en étoiles ${ }^{12}$. Cette présentation des noms de la famille abbasside est très proche de la formule adoptée par Ibn al-Fuwațī dans le dictionnaire biographique dont il sera question ci-dessous. L'auteur, qui semble fortement intéressé par les histoires et les légendes, a préservé beaucoup d'informations d'ordre culturel, en particulier en enregistrant des traditions orales ${ }^{13}$. Au XIII siècle, l'historien persan Minhāğ al-Dīn al-Ǧuzğānī dans ses Țabaqāt-i Nāṣirī signale un texte historique, qui ne nous est pas parvenu, mais qui aurait été composé par Abū l-Qāsim Muhammad al'Imādī. Son titre, Ta'rīh-i muğadwal, indique que cette histoire était sans doute rédigée sous la forme de tableaux ${ }^{14}$.

$\mathrm{Au} \mathrm{XIV}^{\mathrm{e}}$ siècle, on trouve non seulement des taqwìm historiques, mais aussi d'autres types de textes qui utilisent cette présentation. Je désigne ces textes comme un genre littéraire "para-historique": ouvrages géographiques, dictionnaires biographiques, manuels de chancellerie. Il se pourrait qu'à cette époque, au cours de laquelle l'historiographie islamique trouve un important développement, on ait éprouvé le besoin de rationaliser les données pour les rendre plus directement accessibles. Gabrielle M. Spiegel a bien montré dans ses travaux sur l'historiographie en Occident latin que nous devons lire les textes médiévaux en les situant dans le contexte social et culturel dans lequel ils ont pris forme.

8. Le Muğmal al-tawārīh wa l-qișaș est conservé à la Bibiothèque nationale de France (BNF) sous la cote Persan 62. L'identification du nom du grand-père se trouve au fol. 223v. Voir la description codicologique de ce manuscrit par F. RichARD, Catalogue des manuscrits persans I. Ancien fonds, Paris, Bibliothèque nationale, 1989, p. 92-93. Le texte a été édité par Malik al-Šu'rā' Bahār à Téhéran en 1317š/1939. Il existe une édition en fac-similé du manuscrit copié en 751/1350, conservé à la Staatsbibliothek de Berlin, par I. Afshar et M. Omidsalar, Téhéran (Society for Promotion of Persian Culture, Indiana), 2001. La présentation en anglais du texte et de la tradition manuscrite figure dans l'introduction, p. 1-15. On a répertorié au total quatre manuscrits de ce texte : le premier, celui de la Staatsbibliothek de Berlin, est le plus ancien ; le deuxième est celui qui est conservé à Paris à la BNF sous la cote Persan 62, copié en 813/1410 par un certain 'Alī b. Mahmūd ; le troisième est à Dublin à la Chester Beatty Library, il est daté de 823/1420 ; le quatrième enfin, plus tardif, se trouve à la Bibliothèque de Heidelberg, il porte la date de 906/1500, voir l'introduction à la reproduction du texte en fac-similé par I. AfSHAR et M. OMidSALAR, p. 6.

9. Voir les fol. 277v-280r.

10. Le manuscrit a été restauré par l'historiographe ottoman, Muhammad Čelebi Ta‘līq-Zāda (m. 1068/1599), voir F. RICHARD, Catalogue des manuscrits persans I, p. 92.

11. On remarque le manque de rigueur dans la manière d'indiquer le nom des rubriques où l'on trouve un mélange de persan et d'arabe.

12. Tableau des noms et des laqab des prophètes (fol. 277 v-r) ; le Prophète Muhammad, les quatre premiers califes (fol. 278r) ; les Abbassides (fol 278v) ; rois et sultans de l'islam (fol. 279 r) ; Ghaznévides (fol. 279v) ; Seldjukides (fol. 280r).

13. Voir l'introduction à la reproduction du texte en fac-similé par I. Afshar et M. OMidsalar, p. 4.

14. Voir F. RosentHaL, A History of Muslim Historiography, Leyde, Brill, 1968, p. 143. 
Ils sont, en effet, le reflet d'un changement dans la vision de l'histoire ${ }^{15}$. L'adoption de nouveaux canons dans l'historiographie occidentale correspond à des changements sociaux et politiques. Ces schémas d'analyse sont transposables à l'islam. Il semble en effet qu'à cette époque, il y eut une volonté de la part du pouvoir de rendre plus facilement accessible les données historiques en rationalisant la présentation graphique des textes à des fins de propagande politique ou encore pour répondre à la demande d'un public particulier. Avant de présenter une sélection de taqwìm historiques, je donnerai rapidement quelques exemples d'ouvrages scientifiques utilisant une présentation graphique des données.

\section{Quelques taqwìm scientifiques}

Les tables astronomiques

Je commencerai par deux exemples astronomiques ${ }^{16}$. Tout d'abord l'ouvrage d'alZarqālī, al-Qānūn li-Amūnyūs ${ }^{17}$ (Le canon d'Amonius), est un zĭğ établi par un savant de Tolède (m. 1100) qui reprend lui-même un ouvrage grec du III ${ }^{\mathrm{e}}-\mathrm{IV}^{\mathrm{e}}$ siècle ${ }^{18}$. Les chiffres sont donnés en abğad; en effet, entre autres comme chez les Grecs, chaque lettre de l'alphabet arabe avait une valeur numérique en fonction de sa place ${ }^{19}$. L'ouvrage d'al-Zarqālī, connu dans le monde latin sous le nom d'Azarqiel, a bénéficié, dès le $\mathrm{XI}^{\mathrm{e}}$ siècle, d'une traduction latine et, au XIII siècle, d'une autre en castillan sous le roi de Castille Léon Alfonso X, dit le Sage, (r. 1252-1284) ${ }^{20}$, ce qui atteste de sa notoriété en dehors de l'islam. Le second exemple choisi est le Tahrīir al-mağistī (Rédaction de l'Almageste) du savant iranien Nașīr al-Dīn al-Ṭusīi (m. 672/1274) ${ }^{21}$. Il s'agit d'une version en arabe du traité d'astronomie composé $\mathrm{au} \mathrm{II}{ }^{\mathrm{e}}$ siècle par Ptolémée ${ }^{22}$. Les premiers zīğ, rédigés au VIII ${ }^{\mathrm{e}}$ siècle, s'appuient sur la tradition persane et indienne, mais, à partir du IX siècle, la tradition de Ptolémée devint prédominante ${ }^{23}$. L'ouvrage de ce dernier constitua alors la référence la plus importante pour les astronomes musulmans de même que pour les géographes. Nașīr al-Dīn al-Ṭūsī

15. G. M. SPIEGEL, The Past as Text. The Theory and Practice of Medieval Historiography, Baltimore et Londres, The Johns Hopkins University Press, 1997. Sur cette question, voir également P. J. GEARY, La mémoire et l'oubli à la fin du premier millénaire. Traduit de l'anglais par Jean-Pierre Ricard, Paris, Aubier, 1996.

16. Sur l'usage des taqwìm en astronomie, voir M. Hofelich, « Takwīm », EI², vol. X, p. 136-138.

17. Ce manuscrit fut copié en Espagne ou au Maroc en 1267 ; voir L'âge d'or des sciences arabes. Exposition présentée à l'Institut du monde arabe, Paris 25 octobre 2005-19 mars 2006, Arles, Actes Sud/IMA, 2005, p. 110.

18. Voir la reproduction d'un folio du manuscrit in L'âge d'or des sciences arabes, p. 110.

19. Sur l'abğad voir G.S. Colin, « Abdjad », EI $I^{2}$, vol. I, p. 100.

20. Le roi Alfonso X était lui-même féru d'astronomie puisqu'il rédigea des tables astronomiques (Tabulce astronomicce) qui étaient destinées à enrichir, ou à rectifier, les observations de Ptolémée. La version castillane est conservée à la Bibliothèque de l'Arsenal à Paris, voir L'âge d'or des sciences arabes, p. 110.

21. Ce manuscrit est conservé à la BNF sous la cote Arabe 2485, voir en annexe I, fig. 1, la reproduction des fol. 83v$84 \mathrm{r}$.

22. Sur le traité de Ptolémée, voir D. PINGREE, Preceptum Canonis Ptolemei, Louvain-la-Neuve, Bruylant-Academia, 1977.

23. La première traduction de l'Almageste eut lieu en syriaque avant d'intégrer l'astronomie arabe. La littérature syriaque a été un facteur des transferts culturels jusqu'à l'époque ilkhanide incluse, comme en atteste le dernier grand auteur syriaque, Barhebræus. 
dit avoir rédigé ce traité en images pour ses disciples afin de rendre le texte original de Ptolémée plus abordable qu'à travers ses traductions arabes antérieures ${ }^{24}$. Il apporte des corrections et des enrichissements par rapport à ces anciennes traductions. De fait, Nașir al-Dīn al-Ṭūsī rédige une nouvelle version du texte de Ptolémée. Le but affiché ici est donc nettement pédagogique.

\section{L'usage des taqwīm en médecine}

Les manuscrits médicaux sont souvent, du point de vue visuel, rédigés pour faciliter la recherche d'un renseignement précis ${ }^{25}$. Ainsi, la présentation en tableaux appartient à la tradition médicale arabe. C'est, en effet, le médecin du XI siècle, Ibn Buṭlān qui, dans son Taqwìm al-șihha, fut le premier à avoir recours à ce mode de présentation des connaissances médicales ${ }^{26}$. L'ouvrage est dédié à al-Malik al-Ẓāhir al-Ġāzī, le fils de Saladin. L'auteur offre les règles fondamentales d'hygiène et de diététique et se propose d'étudier 280 maux qui peuvent affecter le corps de l'homme en les inscrivant par groupe de 7 dans 40 ğadwal qui comprennent chacun 15 cases ${ }^{27}$. Dans son introduction, Ibn Buṭlān déclare qu'il utilise cette présentation graphique afin de faciliter la consultation de l'ouvrage. Gérard Troupeau a démontré que le terme taqwìm dans le titre de l'ouvrage d'Ibn Buțlān signifie bien « établissement, fixation, détermination » et non pas « redressement » comme Hosam Elkhadem, l'éditeur du texte, a traduit ce terme ${ }^{28}$. Il s'agit d'établir, de déterminer et de fixer la santé. Un second exemple médical confirme également le besoin de présenter les données sous une forme facile à consulter. Il s'agit du Taqwìm al-abdān rédigé par le savant chrétien de Bagdad, Ibn Ǧazla, qui se convertit par la suite à l'islam ${ }^{29}$. L'auteur traite en 44 tableaux de 352 maladies ${ }^{30}$. Comme Ibn Buțlān, il prétend avoir rédigé ce texte à des fins de consultation rapide et facile. Les tableaux établis par Ibn Ǧazla reflètent bien, par leur présentation systématique et d'après les noms retenus pour désigner les différentes rubriques, sa volonté d'adopter une attitude rationnelle face à la maladie : cette dernière a une cause que le médecin doit découvrir rapidement pour guérir le patient.

24. On trouve en marge des annotations explicatives. Ce texte faisait partie d'une collection de manuscrits rapportés d'Orient à la demande de Colbert entre 1671 et 1675, voir L'âge d'or des sciences arabes, p. 82.

25. À l'ombre d'Avicenne. La médecine au temps des califes. Exposition présentée du 18 novembre 1996 au 2 mars 1997, 1996, Paris/Gand, IMA et Snoeck-Ducaju, p. 321.

26. Sur cet auteur voir J. ScнAсHT, « Ibn Buțlān », EI², vol. III, p. 763-764. Il existe une traduction latine de cet ouvrage, intitulée Tacuinum saniatis, qui fut faite au XIII siècle et qui fut publiée à Strasbourg en 1531, voir À l'ombre d'Avicenne, p. 194.

27. Voir la reproduction de deux folios de ce manuscrit, ibid. Ce manuscrit est conservé à la British Library à Londres sous la cote Or. 1347.

28. Le Taqwìm al-Ṣiḥha (Tacuini Sanitatis) d'Ibn Buțlān: un traité médical du XIe siècle, histoire du texte, édition critique, traduction, commentaire par H. Ецкнаdem, Louvain, Peeters, 1990. Voir le compte rendu critique de cette édition par G. Troupeau in Bulletin critique des Annales islamologiques, vol. 10 (1993), p. 194.

29. Sur ce médecin, voir J. Vernet, « Ibn Djazla », EI², vol. III, p. 776-777. Il dédia son ouvrage en 467/1075 au calife abbasside al-Muqtadī (r. 467-487/1075-1094).

30. Voir en annexe I, fig. 2, la reproduction d'un fol. du manuscrit de ce texte conservé à Bagdad, reproduit dans F. MICHEAU, Les Pays d'Islam VII ${ }^{e}$ - XVI ${ }^{e}$ siècle, Paris, La Documentation française, nº 8007, 1999, p. 40. 
À partir de ces quelques exemples relevant des sciences islamiques, on constate que, la plupart du temps, ces ouvrages qui comportent des tableaux ou des éléments graphiques avaient pour but essentiel de rendre les informations rapidement accessibles, comme dans le cas des deux taqwìm médicaux ici présentés, ou qu'ils avaient un but pédagogique, comme la traduction en arabe révisée par Nașīr al-Dīn al-Ṭūsī du traité astronomique de Ptolémée. On peut se poser la question : les ouvrages scientifiques arabes ont-ils eu une influence sur la présentation graphique de l'histoire? Nous allons examiner dans un premier temps des textes proches de l'histoire proprement dite.

\section{Les taqwìm dans la littérature "para-historique" \\ La tradition géographique}

Les ouvrages géographiques se prêtèrent facilement à ce mode de présentation des données de manière visuelle ${ }^{31}$. L'ouvrage le plus célèbre en la matière est le Taqwìm albuldān d'Abū l-Fidā' qui fut achevé en 721/1321 ${ }^{32}$. Il s'agit d'un ouvrage de géographie descriptive complété par des données physiques et mathématiques présentées sous la forme de tableaux. Le texte a fort heureusement été édité en respectant sa présentation manuscrite ${ }^{33}$. À la fin de chaque partie, rédigée sous la forme d'un récit narratif dont le but est de décrire un « pays » (bilād), comme, par exemple, le Bilād al-Šām, l'exemple choisi ici, l'auteur a ajouté des taqwìm. L'objectif de ces tables, qui se présentent un peu sous la forme des zĭğ, est de donner un accès rapide aux coordonnées géographiques (latitudes et longitudes des localités) afin de faciliter l'utilisation de l'ouvrage. Les tableaux sont disposés sur des doubles pages. La page de droite est divisée en dix colonnes qui sont subdivisées en cases ${ }^{34}$. Comme en arabe, la lecture se fait de droite à gauche. La première colonne (satr al-'adad) renferme le numéro d'enregistrement dans le tableau des noms de lieux. La deuxième (al-asmā') recense les noms des villes ou des régions. Dans la troisième colonne (asmā' al-manqūl), l'auteur cite ses sources. Dans les quatrième et cinquième colonnes, il mentionne les degrés de longitude ( $a l-t \underline{u} l)$, avec les minutes (al-daqā’iq) et dans les sixième et septième colonnes, les degrés de latitude (al-'ard), avec les minutes. La huitième colonne (al-iqlìm al-haqia $\bar{l}$ ) comporte les climats astronomiques, tandis que dans la neuvième colonne (al-iqlìm al-'urfï), l'auteur cite le nom de la région par lequel on a coutume de la désigner. Enfin, dans la dixième colonne (dabt al-asmā'), l'auteur précise la vocalisation des toponymes mentionnés dans la deuxième colonne. Sur la page de gauche, partagée en bandes horizontales qui correspondent chacune aux cases de la page de droite, l'auteur donne des informations géographiques plus générales ${ }^{35}$. Abū l-Fidā' s'inspire

31. Voir Islamic aera studies with information systems, O. ATSUYKI (éd), Londres, Routledge Curzon, 2004 ; E.S. et M.H. KENNEDY, Geographical coordinates of localities from Islamic sources, Francfort, Institüt für Geschischite der ArabischIslamwissenschaft, 1987.

32. Voir H. A. R. GIBB, « Abū l-Fidā’ », EI $I^{2}$, vol. I, p. 122.

33. Géographie d'Aboulféda. Texte arabe publié d'après les manuscrits de Paris et de Leyde aux frais de la Société asiatique par M. Reinaud et M. le baron Mac Guckin de SLANE, Paris Imprimerie royale, 1840.

34. Voir en annexe II, fig. 3.

35. Voir en annexe II, fig. 3. 
manifestement dans son Taqwìm al-buldān des zĭğ astronomiques dans le but de permettre au lecteur de localiser facilement les villes et les régions citées dans les tables grâce à ces informations mathématiques et physiques.

\section{L'emploi de tableaux dans les ouvrages biographiques : un cas isolé?}

Nous approchons plus de l'histoire proprement dite lorsqu'on examine le dictionnaire biographique arabe intitulé Talhịș mağma al-ādāb fì mư̆ğam al-alqāb ${ }^{36}$ composé par Kamāl al-Dīn Abū l-Faḍl 'Abd al-Razzāq al-Šaybānī al-Ḥanbalī, plus connu sous le nom d'Ibn al-Fuwați (642-733/1244-1323). Ce dernier fut à la fois bibliothécaire, copiste et auteur d'ouvrages historiques. Il fréquenta les milieux intellectuels d'Azerbaïdjan et la cour de l'Ilkhan Öljeitü (r. 703-717/1304-1317), ce qui lui permit de recueillir de nombreuses données biographiques, en particulier d'ordre culturel ${ }^{37}$. Le manuscrit autographe du volume IV, daté de 712/1312, est conservé à la Maktabat al-Asad de Damas ${ }^{38}$. Très justement Jacqueline Sublet souligne que l'auteur conçoit « une grille qui présente le nom propre arabe médiéval dans sa complexité de manière didactique ${ }^{39}$ ». Ibn al-Fuwați prend en compte non seulement la complexité du nom propre, mais également la hiérarchie de ses composantes ${ }^{40}$. Nous retrouvons ici les préoccupations des auteurs scientifiques dont les ouvrages sous forme de tables auraient pu l'influencer dans son choix graphique tout à fait original.

Ibn al-Fuwațī utilise l'encre rouge pour les tableaux et l'encre noire pour le texte. Lorsqu'on ouvre le manuscrit, on trouve sur la page de droite un double cadre rouge divisé en dix sections horizontales, chaque section comprenant elle-même six cases à l'intérieur desquelles sont notées les informations suivantes: laqab, kunya, ism, filiation, nisba et profession. Sur la page de gauche, sont reportées les mêmes divisions horizontales, et chaque espace ainsi réservé comporte un résumé de la vie du personnage ${ }^{41}$.

Cet exemple atteste du souci de l'auteur de ce célèbre dictionnaire biographique de simplifier la lecture du texte par une présentation graphique des données onomastiques qui permet de distinguer rapidement les différents éléments du nom du personnage évoqué. Ces éléments sont complétés par une courte notice biographique dans laquelle

36. L'auteur avait le projet d'écrire un ouvrage en 50 volumes, contenant toutes les biographies des lettrés depuis les débuts de l'Islam. Conscient que l'entreprise ne pourrait être réalisée, il en fit un abrégé (talhị̂s). Le nom des personnages est classé par laqab "surnom" ou "titre honorifique”, sur ce dictionnaire, voir J. SUBLET, « Dans l'Islam médiéval, nom en expansion, nom à l'étroit : L'exemple d'Ibn al-Fuwați », in L'écriture du nom propre, textes réunis et présentés par A.-M. Christin, Paris, L’Harmattan,1998, p. 117-134.

37. Voir, D. DEWEESE, «Cultural transmission and exchange in the Mongol Empire: Notes from the biographical Dictionnary of Ibn al-Fuwațī », in Beyond the Legacy of Genghis Khan, L. Komaroff (éd.), Leyde, Brill, 2006, p. 11-29.

38. J. Sublet, «Dans l'Islam médiéval », p. 120, note $4:$ «Il s'agit d'un unicum de la quatrième partie (al-ğuz' alrābic) du texte, conservé sous la cote Ta'rih 267 du fonds Zāhiriyya ». Le texte a été édité à Damas (1962-1967) par M. Ğawād, malheureusement sans en respecter la présentation manuscrite

39. J. Sublet, « Dans l'Islam médiéval », p. 117.

40. J. Sublet, « Dans l'Islam médiéval », p. 120.

41. Voir en annexe III, fig. 4, les fol. 229v et 230r du manuscrit conservé à Damas, reproduit dans l'article de J. Sublet. 
sont mentionnés le nom des maîtres du personnage en question, les fonctions dont il a été le titulaire, ses activités intellectuelles, les titres des ouvrages qu'il a pu composer et, souvent, quelques vers de sa composition.

\section{La présentation graphique de certains manuels de chancellerie}

À l'époque timouride, on trouve des recueils de modèles de correspondances présentés sous forme graphique avec des tableaux synoptiques disposés sur plusieurs colonnes, comme le Mahsan al-inšă', composé en 907/1501 par Kamāl al-Dīn Husayn b. 'Alī al-Sabzawārī Wā'iz al-Bayhāqī (m. 910/1504-1504) ${ }^{42}$ qui est dédié au sultan Mu'izz al-Dīn Abū l-Ġāzī Ḥusayn alBayqarā (r. 875-912/ 1470-1506) et à son vizir Mīr 'Alī Šīr Nawā'i ${ }^{43}$. Le manuscrit conservé à la Bibliothèque nationale de France est une copie qui a été achevée en 953/1547 ${ }^{44}$. Outre le choix de cette présentation sous la forme de tableaux des formules destinées à fournir aux scribes de la chancellerie des modèles pour rédiger les lettres, ceci en fonction du statut des destinataires, il faut ajouter que ce manuscrit utilise le trilinguisme, mais il s'agit d'un ajout ultérieur. En effet, avant le début du texte, deux pages ont été ajoutées (fol. 1r-v et fol. 2r). Sur ces folios, des bayt en persan et en turc ont été copiés par au moins deux mains différentes. On note également que le manuscrit a été ensuite repaginé après cet ajout. Il semble donc que des scribes de la chancellerie timouride ont éprouvé le besoin d'ajouter ces vers en langue turque. Il se pourrait que cet ajout de deux folios ait été réalisé au moment de la copie conservée à la Bibliothèque nationale de France, en 953/1547, soit une quarantaine d'années après la rédaction de l'ouvrage.

Ces vers en turc pourraient bien avoir été destinés à illustrer le propos des lettres adressées aux sultans ottomans qui, selon certains chercheurs turcs, sans doute par esprit nationaliste, pensent que les Ottomans avaient une grande admiration pour cette culture chaghatay orientale. Il est vrai que l'alphabet ouïgour semble avoir été en usage à côté de l'alphabet arabe dans les chancelleries ottomanes. Le sultan Murād II (premier règne 824848/1421-1444, second règne 850-855/1446-1451) aurait entretenu à sa cour d'Erdine des secrétaires capables de rédiger des firmān en ouïgour et les princes héritiers se voyaient enseigner cet alphabet ${ }^{45}$. Il ne subsiste cependant qu'un seul document, celui de Mehemmet II (premier règne 848-850/1444-1446, second règne 853-886/1451-1481) qui annonçait aux gouverneurs locaux d'Anatolie orientale sa victoire sur le souverain des Aq Qoyunlu, Uzun Hasan, sous la forme d'un fatḥ-nāma rédigé en alphabet oü̈gour avec une traduction arabe

42. Le Mahsan al-inša' est conservé à la BNF sous la cote Persan 73. Voir la description codicologique de ce manuscrit par F. Richard, Catalogue des manuscrits persans I, p. 101.

43. La dédicace se trouve au fol. 4v. Ce manuscrit est arrivé à la bibliothèque de Colbert le 24 mai 1681, F. RICHARD, ibid. Il en existe une version abrégée, par le même auteur, le Șahîfa-i Šāhī (Suppl. persan 467), ce qui indique que ce texte était utile aux jeunes scribes de la chancellerie timouride. Voir en annexe IV et V, fig. 5 et 6 , les fol. $6 \mathrm{v}$ et $7 \mathrm{v}$ du manuscrit Persan 73 et en annexe VI, fig. 7, le fol. 44v du Suppl. persan 467.

44. La date de la copie, ramaḍān 953, figure dans le colophon au fol. 191v.

45. Sur l'influence du turc chaghatay chez les Ottomans, voir M. F. KöPRÜUÜ, « Osmanlī », partie II, « La littérature », $E I^{2}$, vol. VIII, p. 215. 
interlinéaire ${ }^{46}$. Il se pourrait que le sultan ottoman ait choisi de rédiger ce fath-nāma en utilisant cet alphabet parce que Uzun Hasan lui-même l'employait dans ses firmān, mais à travers la tradition de la chancellerie persano-mongole ${ }^{47}$. Le trilinguisme utilisé dans ce manuel de correspondance atteste de la proximité entre culture turque et persane. Celle-ci remonte aux Qarakhanides qui, au milieu du $\mathrm{X}^{\mathrm{e}}$ siècle, se convertirent à l'islam. Peu à peu, une culture turco-persane fit ainsi son apparition dans laquelle l'élément turc trouva naturellement sa place. L'exemple le plus célèbre est celui de Yūsuf Ḩāṣṣ Ḥāğib qui adapta à la langue turque les canons de la littérature persane. En 462/1069, il acheva à Kashghar un long poème didactique en turc oriental, dans la tradition des "Miroirs des princes persans », le Qutadgu Bilig (La sagesse qui conduit à la gloire royale), rédigé sur le même mètre que le Livre des rois de la Perse antique, le célèbre Šāh-nāma de Firdaws $\overline{1}^{48}$. Cette proximité culturelle se concrétise ici à travers les modèles poétiques proposés dans le Mahzan al-inša' aux scribes de la chancellerie timouride.

\section{L'histoire sous forme graphique ou de taqwìm}

\section{La combinaison entre textes narratifs et arbres généalogiques}

Au préalable, je voudrais m'arrêter un moment sur une forme particulière de présentation de l'histoire qui combine à la fois arbres généalogiques et textes narratifs ${ }^{49}$. Je commencerai en présentant le Mu izz al-ansāb, rédigé sous la forme d'une histoire graphique, qui présente la généalogie de Chinggis Qan et de Tamerlan ainsi que leur descendance respective ${ }^{50}$. Le texte, rédigé en persan, fut composé par un auteur anonyme en 830/1428 sur l'ordre du sultan timouride Šāh-Ruh (r. 807-850/1405-1447). Dans la préface, l'auteur du Mu'izz al-ansāb écrit qu'on lui a demandé de compiler les généalogies existantes afin de produire celle de la lignée de Tamerlan ${ }^{51}$. En effet, le $M u$ uizz al-ansāb apparaît comme une continuation du Šu 'ab-i Panğgānah de l'historien Rašīd al-Dīn qui fut également ministre des Ilkhans Ġazan Qan (r. 694-703/1295-1304) et Öljeitü. Ce texte est une histoire dynastique des Mongols, mais également des Francs, des Chinois, des Arabes et des Juifs ${ }^{52}$. Bien que le Mu'izz al-ansāb soit rédigé sous la forme d'un manuscrit (codex), il est écrit verticalement de manière à imiter la disposition d'un rouleau. Des traits de différentes couleurs marquent

46. Sur ce fath-nāma, voir R.R. ARAT, « Fatih Sultan Mehmed'in yarlı̆ı », Tükiyat Mecmuasi, vol. 6 (1936-1939), p. 285$322+20$ p. du document en fac-similé. Voir en annexe VII, fig. 8 un extrait de ce document, p. 2-3.

47. Sur cette question, voir A. Soudovar, « The Mongol Legacy of Persian Farmāns », in Beyond the Legacy of Genghis Khan, p. 407-421.

48. Yūsuf Khāșș Hājib, Wisdom of Royal Glory [Qutadgu Bilig]. A Turko-Islamic Mirror for Princes, translated, with an Introduction and Notes by R. DANKOFF, Chicago/Londres, The University of Chicago, 1983.

49. Les arbres généalogiques dans l'Islam remontent à une époque beaucoup plus ancienne, mais ils n'inséraient pas de textes narratifs.

50. La copie conservée à la BNF sous la cote Persan 73 est non datée, mais elle fut sans doute rédigée au xvi ${ }^{\mathrm{e}}$ siècle, voir F. RICHARD, Catalogue des manuscrits persans I, p. 97. Il existe une autre version de ce texte à la British Library.

51. S.A. QuinN, «The Mu'izz al-Ansāb and Shu'ab-i Panjgānah as Sources for the Chaghatayid Period of History: A Comparative Analysis », Central Asiatic Journal, vol. 33/3-4 (1989), p. 231.

52. Le Šu'ab-i Panğgānah utilise le bilinguisme: les noms sont écrits à la fois en alphabet arabe et en écriture ouïgoure, S.A. QuinN, « The Mu'izz al-Ansāb and Shu'ab-i Panjgānah », p. 233. 
la descendance des Qan mongols et des sultans timourides. Des emplacements circulaires, préparés pour recevoir les portraits des principaux souverains, ont été réservés, mais malheureusement ils n'ont pas été peints ${ }^{53}$. Dans différents endroits de chaque folio se trouvent consignées des informations sur le règne du Qan en question ${ }^{54}$. Mais ce qu'il faut souligner c'est que de chaque côté de la figure du souverain sont disposées des informations intéressantes par rapport aux choix fait par l'auteur anonyme de ce texte. En effet, du côté droit, on trouve les biographies des grands émirs et du côté gauche les biographies des femmes du Qan. Cette présentation met donc en valeur ces deux catégories de personnes. Dans le monde turco-mongol, les émirs détenaient sans conteste un pouvoir important, mais les femmes des Qan jouaient également un rôle politique considérable. Elles assuraient la régence lors de la vacance du pouvoir et, au moment de l'élection d'un nouveau Qan, elles influençaient l'orientation politique de leurs époux ${ }^{55}$. Le but du Mu'izz al-ansāb est manifestement politique. En commanditant cet ouvrage, Šāh-Ruh voulait faire des Timourides les héritiers de l'Empire créé par Chinggis Qan, mais on peut y déceler également la volonté de la part de l'auteur de rationaliser ou de résumer des données historiques très abondantes sur les lignages mongols et timourides en consignant par écrit l'essentiel de l'histoire complexe de ces deux grands empires sous une forme visuelle graphique, facile à consulter.

À cette même période, XIII-XIV ${ }^{\mathrm{e}}$ siècle, apparaissent des chroniques versifiées selon le modèle du Šāh-nāma de Firdawsī. Plusieurs de ces textes ont été rédigés à l'époque ilkhanide, comme, entre autres, le Šāhanšāh-nāma d'Aḥmad al-Tabrīzī ${ }^{56}$, composé à la gloire de Chinggis Qan et de ses successeurs, le Ġāzān-nāma, d'un auteur anonyme ${ }^{57}$, ou encore le Zafar-nāma de Hamd Allāh Mustawfī al-Qazwīnī ${ }^{58}$, une chronique islamique versifiée avec dans les marges le texte du Šāh-nāma de Firdawsī. Il existe sans doute une relation entre ces histoires versifiées qui ont des effets auditifs et les histoires graphiques qui, elles, ont des effets visuels : dans les deux cas, il s'agit d'une méthode qui facilite la mémorisation tout comme les poèmes mnémotechniques de cette même époque. C'est une manière de donner des informations historiques et d'instruire sous une forme qui facilite la mémoire.

53. Dans le manuscrit conservé à la British Library, les portraits ont été peints, voir S. A. QuinN, « The Mu'izz alAnsāb and Shu'ab-i Panjgānah », p. 233, note 17.

54. Voir en annexe VIII, fig. 9, le fol 13v du manuscrit conservé à la BNF sous la cote Persan 73 (La descendance de Chinggis Qan).

55. Sur le rôle des femmes en Asie intérieure et en particulier à l'époque mongole, voir D. SinoR, « Some Observations on Women in Early and Medieval Inner Asian History », in The Role of Women in the Altaic World. Permanent International Altaistic Conference, 44th Meeting, Walbergberg, 26-31 August 2001, V. VeIt (éd.), Wiesbaden, Harrassowitz Verlag, 2007, p. 261-268.

56. Le manuscrit est conservé au British Museum sous la cote OR 2780, fol. 41-132, voir C. RIEU, Supplement to the catalogue of the Persian MSS in the British Museum, Londres, 1895, p. 135.

57. Ġāzān-nāma, M. TADBBŪRĪ (éd.), Téhéran, 1381š.

58. Le manuscrit est conservé au British Museum sous la cote OR 2833, fol. 779 fol, voir C. RIEU, Supplement to the catalogue of the Persian MSS, p. 172-174. Voir également A. SOUDOVAR, « The saga of Abu-Sa'id Bahādor Khān. The Abu-Sa id nāmé », in The court of the Il-khans, J. RABY et T. FITZHERBERT (éd.), Oxford, Oxford University Press, 1996, p. 95-218. 
On ne peut que rapprocher l'apparition de ces chroniques persanes versifiées du développement, à la même époque en France, d'une historiographie en langue vernaculaire qui avait pour objectif de revivifier la memoria du passé. Comme Gabrielle M. Spiegel l'a bien fait remarquer, la culture médiévale était, dans un certain sens, une « culture de la commémoration » qui passait par la récitation orale sur un mode épique ${ }^{59}$, tout comme le Šāh-nāma de Firdawsī qui était déclamé à la cour des princes par des "déclamateurs de šāhnāma" (̌̌āh-nāmahwwānd) et, par la suite, les chroniques versifiées sur l'histoire des Mongols. Rédiger à la gloire de Chinggis Qan, des Ilkhans Ġāzān ou Abū Sa'īd de telles oeuvres était une manière d'intégrer ces derniers, issus du monde de la steppe, le Tūrān de l'histoire épique de l'Iran antique, à l'histoire et à la culture persane.

De même, cette présentation "graphico-généalogique" de l'histoire est comparable à un phénomène analogue qui apparaît en Europe, toujours à cette même période (XIII-XIV s.). En effet, à partir du dernier tiers du XIII ${ }^{\mathrm{e}}$ siècle, plus particulièrement sous les règnes d'Édouard I ${ }^{\text {er }}$ (1272-1307) et d'Édouard II (1307-1327), on assiste en Angleterre à la floraison d'un nouveau type de chroniques abrégées, centrées autour d'un schéma généalogique ${ }^{60}$. En France à cette même époque des généalogies sont introduites dans le récit historique narratif. La rédaction latine de la Chronique abrégée (1285-1300) de Guillaume de Nangis fut composée sous la forme d'un arbre généalogique avec un tronc et des ramifications ${ }^{61}$. Ce qui est intéressant à souligner, c'est que l'auteur précise dans l'introduction que, considérant que l'histoire des rois de France est prolixe, il a choisi de la rédiger sous la forme d'un arbre généalogique parce qu'il est plus facile de mémoriser par les yeux :

«Considerans hystorie regum Francorum prolixitatem... temptavi seriem cunctarum hystoriarum de ipsis loquentibus dub quidam arboris formula redigere... propter subjectam oculis formam, sit oblectatio, et studiosis facile possit prehabita pre oculis memorie commendari ${ }^{62}$."

On retrouve ici les préoccupations de l'auteur du Mu'izz al-ansāb, même si celles-ci ne sont pas clairement exposées dans l'introduction de son ouvrage, comme le fait Guillaume de Nangis.

Ces histoires généalogiques, comme le souligne Gabrielle M. Spiegel, sont avant tout écrites pour légitimer le pouvoir d'une famille noble ou pour affirmer un pouvoir politique ${ }^{63}$. En d'autres termes, le but est d'exprimer par le graphisme la mémoire sociale d'un groupe, ce qui est le cas du Šu'ab-i Panğgānah et du Mu'izz al-ansāb que l'on peut comparer aux généalogies royales occidentales. Les auteurs cherchent à imposer aux futures générations une représentation sociale des différents clans qui constituaient les lignées princières mongoles et timourides. La généalogie, présentée sous la forme où

59. G.M. SPIEGEL, « Social Change and Literary Language. The textualization of Past in Thirteenth-Century Old French Historiography », in The Past as Text, p. 178-194, en particulier, p. 181-184.

60. Voir en annexe IX, fig. 10, la reproduction d'un fragment d'une chronique anglaise anonyme, conservée à Berlin Staatsbibliotek, Hdschr. 343, reproduite in D. WeLtecKE, Die «Beschreibung der Zeiten» von Mōr Michael dem Grossen (1129-1199), p. 187.

61. Manuscrit conservé à la BNF sous la cote Latin 6184.

62. Cette citation se trouve en introduction au volume au tout début du fol. 1r.

63. G.M. SPIEGEL, « Genealogy. Form and Function in Medieval Historiography », in The Past as Text, p. 104. 
graphisme et textes se combinent, semble avoir eu un impact sur la manière de façonner la narrativité historique tant du côté occidental qu'oriental, en particulier dans le monde turco-iranien. On trouve dans ces différents ouvrages une combinaison entre des éléments graphiques, iconographiques et des textes. Il s'agit de présenter sous la forme d'un schéma commenté les informations historiques qui, sous cette forme symbolique et synthétique, apparaissent avec plus de clarté pour le lecteur. Par conséquent, leur impact pour exprimer la revendication sociale d'une famille noble ou la légitimité politique d'un pouvoir dynastique est directement apparent.

\section{Chroniques historiques et ğadwal}

Passons maintenant à quatre textes historiques qui, eux, combinent texte narratif et ğadwal, à l'exception d'un seul qui est entièrement rédigé sous forme de tableaux ou, pour être plus exact, de "cases". Le premier exemple est une histoire universelle en persan, le Muntahab al-tawärīh. Elle fut terminée en 816/1413 par l'historien timouride Mu'īn al-Dīn al-Națanzi ${ }^{64}$. Ce texte a la particularité de terminer l'histoire de chaque règne, rédigée en prose, par des tableaux synoptiques dynastiques (ğadwal). Cette chronique comporte vingthuit ğadwal, comptant chacun de quatorze à trente rubriques ${ }^{65}$. Il s'agit de la part de $\mathrm{Mu}^{\text {'inn }}$ al-Dīn al-Națanzī d'un véritable effort pour synthétiser les informations historiques. Non seulement l'auteur présente les souverains, les faits marquants et les dates importantes de leur règne, mais il trace surtout leur portrait individuel. La plupart des informations concernent la "lignée" de chaque souverain (laqab, nisba, kunya, nom du père, de la mère, nombre d'enfants, de femmes) et les éléments de sa "vie publique officielle" en tant que souverain (œuvres, grands de son époque, qādì, amìr al-umarā', ministres, dates d'intronisation, durée du règne, date et cause de la mort, lieu de sépulture) ${ }^{66}$.

Cependant, l'originalité des tableaux réside dans le groupe de rubriques qui est censé décrire la "personnalité" des membres du lignage : qualités, actions marquantes, habitudes, talents. Ces tableaux donnent un éclairage spécifique sur la manière dont un lettré persan timouride percevait les familles princières des siècles antérieurs à son époque. Ces rubriques plus "personnelles" se répartissent en trois ensembles : d'abord, sïrat; puis ațvār et piša ; ensuite 'ādat; et, enfin, hunar. Le terme sìrat contient l'idée d'une vie et d'une conduite exemplaires. Dans les tableaux, cet intitulé semble associé à la vision historique qu'il faut retenir du personnage: ses qualités, mais aussi ses défauts comme

64. Sur cet auteur, voir l'introduction de J. Aubin à son édition partielle de cette chronique, Extraits du Muntakhab altavarikh-i Mu'ini (Anonyme d'Iskandar), J. Aubin (éd.), Téhéran, Librairie Khayyam, 1957, p. 1-8. Ce texte a connu deux rédactions différentes en raison de rivalités politiques entre un petit-fils de Tamerlan, Sultān-Iskandar, qui régnait à Chiraz, et son oncle Šāh-Rūh. Il existe à la bibliothèque de Topkapi Saray une histoire anonyme, avec des tableaux, qui fut rédigée en 816/1413 au moment où Sulțān-Iskandar détenait le pouvoir dans le Fārs, voir W.M. THACKSTON, "Anonymous Synoptic account of the House of Timur ", in A Century fo Princes. Sources on Timurid History and Art. Selected and Translated by W. M. Thackston, Cambridge Massachusetts, The Aga Khan Program for Islamic Architecture, 1989 , p. 237-246.

65. Le manuscrit est conservé à la BNF sous la cote Suppl. persan 1651. Voir en annexe X, fig. 11, fol. 12 (Les rois mythiques de la Perse antique, les prophètes et les sages de leur époque).

66. Voir en annexe XII, fig. 12, la traduction du fol. 329v : Ǧadwal de Malik Čūpān Kučik et de ses descendants. 
prince ${ }^{67}$. Les deux intitulés ațvār et piša présentent le "visage public" du souverain et le bilan de son action ${ }^{68}$. Le terme atvār exprime la manière de se comporter, tandis que piša évoque la profession, le métier, l'occupation à laquelle s'adonne un homme et par laquelle il peut tirer sa subsistance ${ }^{69}$. Mu'īn al-Dīn al-Națanzī emploie ces deux termes pour présenter l'œuvre, l'occupation favorite des souverains et de leur descendance. Le terme 'àdat est généralement utilisé pour désigner les habitudes d'une personne. Sous cet intitulé, $M u$ 'īn al-Dīn al-Națanzī présente le personnage dans sa "vie privée" et ses "inclinations personnelles". Le terme hunar, quant à lui, désigne les qualités acquises par opposition aux qualités innées (al-ğawhar) ${ }^{70}$. Sous l'intitulé hunar, Mu'īn al-Dīn al-Națanzī décrit les goûts et les talents des membres du lignage. Les rubriques 'ādat et hunar servent à donner une "image privée" du personnage, parallèlement au contenu des rubriques ațvār et piša qui explicitent son comportement "public".

À partir de la période mongole, les rubriques dans lesquelles $\mathrm{Mu}$ īn al-Dīn al-Națanzī décrit la personnalité des personnages sont plus détaillées et plus nombreuses. Il signale diverses constructions entreprises par les membres de ces lignées princières d'origine nomade. Ananda Musalmān Hुān [sic] se voit attribuer la construction « de mosquées et d'écoles mobiles dans le camp impérial ${ }^{71}{ }^{\prime}$ (masāğid va madāris dar urdū sāhnt). Mu'īn al-Dīn al-Națanzī confond ici Ananda avec son fils, Ōrūk-Tīmūr [Örug Temür], le roi des Tangut de Sibérie qui, lui, s'était converti à l'islam. Avec l'un de ses émirs, il avait fait établir des «tentes mosquées » dans son camp ${ }^{72}$. Bien que l'auteur du Muntahab al-tawārīh se trompe sur l'identité exacte de ce prince, il retient de ce personnage le fait qu'il a adapté au mode de vie nomade deux éléments de la culture musulmane : la mosquée et l'école d'enseignement religieux.

Les tableaux du Muntahab al-tawārīh présentent également l'intérêt de fournir des indications culturelles qui ne se trouvent pas dans le texte de la chronique. Parmi les traits culturels cités, nombreux sont ceux qui portent sur l'écriture, mais les seules références à l'écriture mongole se trouvent dans le tableau consacré aux Chaghatay, dont le khanat est considéré comme celui dans lequel la coutume et la loi mongoles ont perduré le plus longtemps. Čağțāy, le fils de Chinggis Qan, est mentionné dans le tableau pour avoir renforcé « la loi mongole ${ }^{73} »(y \bar{a} s \bar{a})$. Au total, nous trouvons à propos des Chaghatay, cinq références

67. Cette rubrique n'est jamais omise, sauf pour les Qan de la Horde d'or.

68. Ces deux rubriques ne se côtoient jamais dans un même tableau sauf dans celui qui est consacré aux Omeyyades où l'auteur associe les deux termes mais dans une seule rubrique (ațvār va pǐša).

69. Voir Ch.-H. DE Fouchécour, Moralia. Les notions morales dans la littérature persane du $3^{e} / 9^{e}$ au $7^{e} / 13^{e}$ siècle, Paris, Éditions Recherche sur les civilisations (Synthèse n²3), 1986, p. 186.

70. Voir Ch.-H. De Fouchécour, Moralia, p. 207. Pour une définition complète de ces notions, cf. le dictionnaire de 'A. Dihhudā, Luġat-nāma, Téhéran, Mu’assasa-i Dihhuudā, s.d. : ațvār, vol. II, p. 2754 ; pīša, vol. IV, p. 59935995- ; sīrat, vol. IX, p. 13883 ; 'àdat, vol. X, p. 15655 ; hunar, vol. XV, p. 23567.

71. Mu'īn al-Dīn al-Națanzī, fol. $384 \mathrm{r}$.

72. Un manuscrit enluminé du Ǧāmi‘ al-tawārīh de Rašīd al-Dīn, conservé à la BNF sous la cote : Suppl. persan 113, comporte au fol. $139 \mathrm{v}$ une représentation d'une séance de lecture du Coran dans une telle école religieuse mobile dans son camp.

73. Mu'īn al-Dīn al-Națanzī, fol. 265r. Cette mention se trouve dans le tableau consacré à Chinggis Qan et à ses fils. 
à l'écriture turque, cinq à l'écriture persane et celle qui concerne l'écriture mongole : ŠāhTemür (760/159) « écrivait bien en mongol ${ }^{74}$ » (hatțt-i mugiūl nìkū nivištì). Parmi les talents $(\bar{a} \bar{a} b)$ que doit posséder tout homme cultivé ${ }^{75}$, plusieurs sont cités par Mu'īn al-Dīn alNațanzī : deux fois les échecs, trois fois la musique, six fois la poésie. Il présente ainsi les Turco-Mongols, et en particulier les Chaghatay, comme des lettrés, tout en mentionnant également des talents traditionnels chez les nomades: la chasse ( 3 mentions) et le lancement des flèches (également 3 mentions).

On constate que dans les tableaux du Muntakhab al-tawārīh, les souverains et les membres de leur lignage sont davantage décrits selon leurs qualités personnelles que selon leurs actes de gouvernement. Ils sont gratifiés des qualités du prince idéal des Miroirs: justice, savoir et sagesse ${ }^{76}$. Cette histoire universelle, comme le Mu'izz al-ansāb, appartient à l'historiographie timouride. Tamerlan, le fondateur de la dynastie, s'appuie à la fois sur la tradition turco-mongole et sur l'islam. Le conquérant timouride se rattacha à la lignée de Chinggis Qan en se présentant comme le protecteur du khanat chaghatay dont il envahit la partie orientale en rabí ${ }^{`}$ II 761/février-mars 1360. Tamerlan choisit alors un Qan gengiskhanide fantoche, dont le nom fut prononcé dans la huțba du vendredi et qui figurait sur ses monnaies et correspondances diplomatiques ${ }^{77}$. Il prit pour épouse Saray Mulk Qatun, une femme d'ascendance gengiskhanide, revendiquant ensuite le titre de gendre impérial (güregen) ${ }^{78}$. Il se présentait ainsi comme le tenant de l'ordre chaghatayide. Les gestes politiques mis en œuvre par Tamerlan pour justifier sa prise de pouvoir dans le khanat chaghatay expliquent que $\mathrm{Mu}^{\text {tìn }}$ al-Dīn al-Națanzī valorise cette dynastie, mais aussi qu'il dévalorise les Qan de la Horde d'or avec lesquels Tamerlan était en conflit, en particulier avec Toqtamiš ${ }^{79}$. Le dernier tableau de la chronique, consacré à Tamerlan et à ses fils, le présente comme un souverain sage (hakìm), longanime (halìm) et un combattant de la foi $(\dot{g} \bar{a} z \bar{\imath})$. Tamerlan est ainsi décrit par rapport à son attitude comme musulman exemplaire : « il avait un respect exceptionnel pour la loi religieuse » (dar šarīat mubālaǵat $m i-n a m \bar{u} d)$ et « il aimait les descendants du Prophète ${ }^{80}$ ».

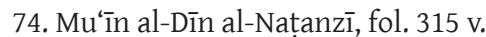

75. Voir Dj. Khaleghi-Motlagh, « Adab », EIr, vol. I, p. 433-435.

76. Voir une vue synthétique sur les Miroirs des princes in D. AIGLE, « La conception du pouvoir en islam. Miroirs des princes persans et théories sunnites (XI ${ }^{\mathrm{e}}$-XIV siècles) », Perspectives médiévales, vol. 31 (2007), p. 17-44, où l'on trouvera une abondante bibliographie récente sur le sujet. Voir en annexe XII, fig 15, le tableau récapitulatif des appréciations de Mu'īn al-Dīn al-Națanzī sur les lignées turco-mongoles.

77. Sur la recherche de légitimité gengiskhanide, voir B. Forbes MANZ, «Tamerlane and the Symbolism of Sovereignty ", Iranian Studies, vol. 21/1-2 (1988), p. 105-122 ; idem, The Rise and Rule of Tamerlane, Cambridge, Cambridge University Press, 1989 ; idem, « Tamerlane's Career an its Uses », Journal of World History, vol. 13/1 (2002), p. 1-25.

78. Güregen est un terme mongol qui désigne le gendre et, à l'époque gengiskhanide, le mari d'une princesse impériale, voir P. D. BUELL, Historical Dictionary of the Mongol World Empire, Lanham, Maryland et Oxford, The Scarecrow Press 2003, p. 159.

79. Sur les rivalités entre Tamerlan et Toqtamiš, voir B. ForBes MANZ, « Mongol History rewritten and relived ", in Figures mythiques des mondes musulmans, D. AIGLE (dir.), Revue des mondes musulmans et de la Méditerranée, vol. 89-90 (2000), p. 129-149.

80. Mu'în al-Dīn al-Națanzī, fol. 363 r. 
Après avoir décrit Tamerlan comme un souverain musulman parfait, Mu'īn al-Dīn al-Națanzī attribue à ses fils des qualités plus spécifiques aux nomades : ils sont braves (bahādur) et généreux (sahī). La lignée de Tamerlan s'inscrit en continuité, à travers les Qan du khanat mongol des Chaghatay, avec celle de Chinggis Qan, mais dans un cadre strictement musulman. De fait, Tamerlan et ses fils s'inscrivent en contrepoint de la présentation d'ensemble des princes turco-mongols de la période précédente ${ }^{81}$. Les Timourides se rattachent par les qualités qui leur sont attribuées à la tradition des Miroirs des princes islamiques et au monde nomade : bravoure et générosité. La présentation nuancée des souverains turco-mongols valorise implicitement la dynastie timouride. Cependant, dans les tableaux, que l'on peut considérer comme des "documents pédagogiques", Mu'īn alDīn al-Națanzī présente la dynastie timouride selon un aspect idéalisé. En effet, dans le récit qu'il donne du règne de Tamerlan, il écrit que les émirs lui avaient souhaité d'obtenir d'autres victoires et ce dernier aurait déclaré que « le décret céleste et la loi gengiskhanide » (yarlìg-i āsmānī va tūra-yi čingīzhānī) lui donnaient le droit à régner ${ }^{82}$. En d'autres termes, bien que se présentant comme un musulman exemplaire, le conquérant timouride n'avait en aucun cas abandonné sa culture turco-mongole.

\section{L'histoire en "cases"}

Un autre manuscrit persan, anonyme, qui, d'après l'écriture, pourrait dater du XVIII ${ }^{e}$ siècle, mais qui a pu être rédigé un peu plus tôt, se présente entièrement sous forme de ğadwal ${ }^{83}$. Cependant, les rubriques sont beaucoup plus rudimentaires par rapport à celles qui avaient été choisies par Mu'īn al-Dīn al-Națanzī. Il s'agit plutôt de "cases" dans lesquelles l'auteur a consigné des informations très brèves, pas toujours exactes d'ailleurs, sur les souverains évoqués : nom, date d'intronisation, durée du règne. Cependant, du point de vue culturel, ce qui est intéressant à relever ici, c'est que l'auteur a pour objectif de présenter sous cette forme une véritable histoire du monde, au sens propre du terme. Il a pu être influencé par le Ǧāmi' al-tawārîh de Rašīd al-Dīn qui, à la demande de l'Ilkhan Ġazan Qan, puis de son successeur, Öljeitü, a composé un ensemble d'histoires consacrées aux Mongols, aux Turcs Öguz, aux Indiens, aux Chinois, aux Juifs et aux Francs ${ }^{84}$. En effet, l'auteur anonyme de ce taqwìm historique consacre, lui aussi, des tableaux à des peuples non musulmans comme les rois de Chine, les anciens Turcs, les Mongols, les souverains de l'Inde, les Pharaons d'Égypte, les rois des Juifs, les Francs, les Anglais, etc. Ce texte n'a absolument aucune valeur historique, mais il témoigne de la part de son auteur d'une rare curiosité d'esprit et d'un important effort pour collecter toutes ces informations sur des

81. Voir, en annexe XII, fig. 13.

82. Mu'īn al-Dīn al-Națanzī, Extraits du Muntakhab al-tavarikh-i Mu'ini, J. AUBIN (éd.), Téhéran, 1957, p. 206.

83. Ce manuscrit est conservé à la bibliothèque de l'Institut français de recherche en Iran sous la cote Ms. 18.

84. Une partie des histoires de Rašīd al-Dīn consacrées à des peuples non mongols a été récemment éditée pour la première fois, voir Rashīd al-Dīn Faẓl Allāh Hamadānī, Jāmi' al-Tavārīkh (The History of Afranj, Popes and Caesars), M. Rushan (éd.), Téhéran, Mīrāt-i Maktūb, 2005 ; idem, Jāmi al-Tavārīkh (The History of India, Indus and Cashmere) ; idem, Jāmi' al-Tavārīkh (History of Ughūz). L'édition de ces trois textes de Rašīd al-Dīn vient utilement compléter l'édition de son Histoire de la Chine, History of China and Cathay. Being a fragment of Jāmit at-Tawārīkh, W. YIDAN (éd.), Téhéran, Iran University Press, 2000. 
DENISE AIGLE

peuples si divers. Comme dans le cas des textes non historiques présentés sous la forme de ğadwal, il s'agit pour ce lettré persan anonyme de donner un accès rapide à un grand nombre d'informations sur l'histoire du monde depuis l'histoire biblique jusqu'à l'époque de la rédaction de l'ouvrage.

\section{Les taqwīm historiques ottomans}

Pour terminer l'inventaire de ces textes historiques graphiques ou combinant tableaux, généalogies et textes narratifs, j'examinerai deux chronographies d'époque ottomane qui furent rédigées sous la forme de taqwìm, mais qui incluent des commentaires textuels dans les tableaux et dans les marges. La partie ğadwal de ces histoires est précédée d'une introduction (muqaddima) dans laquelle les auteurs expliquent les différentes façons de compter les temps depuis Adam ${ }^{85}$. Ils citent le nom des mois chez les Arabes, les Syriens, les Persans, etc. On y trouve une explication sur le calendrier ğaläl ${ }^{86}$, instauré au XI ${ }^{\mathrm{e}}$ siècle par le souverain seldjoukide Ǧalāl al-Dīn Malik Shāh, à l'instigation d'un groupe d'astronomes persans. Ils donnent également des explications sur le calendrier sino-ouïgour utilisé par les Turco-Mongols qui, lui, repose sur un cycle de 12 années désignées par le nom d'un animal ${ }^{87}$.

Le premier texte d'époque ottomane examiné ici est intitulé: Taqwīm al-tawārīhn ${ }^{88}$. Il s'agit d'une chronographie sous forme de tables qui débute avec la création d'Adam et s'achève en 1058/1648, date à laquelle le texte fut terminé par Hāāğğì Halīfa, connu

85. On remarque que l'auteur anonyme de la seconde chronographie s'est contenté de recopier mot à mot l'introduction de Kātib Čelebi.

86. Le calendrier ğalālī ou malikī avait pour but de déterminer la date de nōrūz. Elle fut fixée à l'équinoxe de printemps, moment où le soleil entre dans le Bélier, ce qui correspondait au 15 mars du calendrier julien; les mois étaient de trente jours selon la tradition persane. Ce calendrier servit de base pour les calendriers agricoles en Perse jusqu'à un passé récent, voir B. van DALEN « Ta'rīkh », $E I^{2}$, vol. X, p. 287.

87. Ce calendrier fut introduit en Iran sous la dynastie des Ilkhans, le plus ancien zĭg de cette nature est le Zĭg-i îlhānī du savant ismaélien Nașîr al-Dīn al-Ṭūsī ; il est semblable au calendrier solaire-lunaire, le Ta Ming Li, adopté par Chinggis Qan après sa conquête de la Chine du Nord en 1215, voir R. MERCIER, « The Greek 'Persian Syntaxis' and the Zij-i Ikhānī », Archives internationales d'histoire des sciences, vol. 34 (1984), p. 33-60 ; Ch. Melville, « The Chinese Uighur Animal Calendar in Persian Historiography of the Mongol Period », Iran, vol. 32 (1994), p. 83-98 et Th. T. Allsen, Culture and Conquest in Mongol Eurasia, Cambridge, Cambridge University Press, 2001, le chapitre « Astronomy », p. $161-175$

88. Le manuscrit est conservé à la BNF sous la cote Suppl. persan 1739. Selon É. Blochet, ce manuscrit est sans doute une copie que Kātib Čelebi aurait fait exécuter pour son propre usage. Le scribe en effet a laissé des pages en blanc destinées à recevoir des additions ultérieures. Une personne en possession de laquelle passa ce manuscrit a ajouté la date de l'avènement des Ottomans jusqu'à Sulțān Maḥmūd en 1223/1808. On peut lire sur les dernières pages d'une main du xvIII ${ }^{e}$ siècle un petit poème en turc sur la géomancie et la liste des gouverneurs de Bosnie depuis 867/1262-1463. Le manuscrit fut copié à Istanbul au milieu du XVII siècle. Il comporte 126 feuillets. Voir É. LOCHET, Catalogue des manuscrits persans, vol. IV, Paris, Bibliothèque nationale, p. 239. Il existe à la BNF d'autres textes de ce type, tous inspirés par le Taqwīm al-tawārīh de Kātib Čelebi comme, par exemple le Suppl. turc 921 : «Chronologie abrégée des empereurs turcs, des vizirs, des muftis depuis la fondation des Ottomans ». Le texte comporte 38 feuillets et s'arrête avec le règne du sultan Mahmūd I Ir ; il date de la première moitié du XVIII ${ }^{\mathrm{e}}$ siècle. Le Suppl. turc 925 est presque identique. Ces deux textes ont été traduits en français par Joseph Wiet (jeune de langues de France à Constantinople, en 1742, voir É. BLOCHET, Catalogue des manuscrits turcs, Paris, Bibliothèque nationale, MDCCCXXXIII p. 96-98. 
également sous le nom de Kātib Čelebi (1017-1067/1609-1657) ${ }^{89}$. L'auteur précise dans une autre de ses œuvres, le Mīzān al-haqq fì ihtiyār al-ahaqq, que ce texte a été écrit pour servir d'index à sa grande histoire universelle en arabe, la Fadalakat al-tawārīh rédigée en 1051/1641 ${ }^{90}$. L'introduction (muqaddima) et la conclusion (hatima) de ce taqwim sont en ottoman, le contenu des tableaux est rédigé en persan, mais les dates chiffrées sont également transcrites en écriture turque. Le bilinguisme qui caractérise ce texte est une nouvelle illustration du fait que nous sommes ici à la jonction entre les mondes persan et turc qui étaient, comme on l'a vu, culturellement proches de longue date.

Le Taqwīm al-tawārīh de Kātib Čelebi a eu un rayonnement important puisque plusieurs auteurs ottomans ont rédigé des textes sur ce même modèle, mais cette tradition s'arrête à la fin du XIX ${ }^{e}$ siècle avec une histoire d'Ibrāhīm Aġāh Paša qui est encore présentée sous cette forme ${ }^{91}$. Kātib Čelebi voulait sans doute aider les lecteurs de son histoire universelle arabe en prose en leur procurant une sorte d'index, rédigé sous la forme de tableaux ${ }^{92}$. Le but ici est encore d'ordre pédagogique : simplifier une histoire complexe en en donnant un résumé rapide et synthétique qui permet de se reporter ensuite à la " grande chronique » afin que celui qui souhaite s'informer plus amplement sur certains moments de l'histoire puisse s'y référer facilement.

Le Taqwīm al-tawārīh de Kātib Čelebi présente en son début des éléments de canons chronologiques, mais très simplifiés par rapport aux véritables zĭğ. Par ailleurs, les rubriques ne comportent aucun trait qui pourrait qualifier les personnages, ni aucun élément d'ordre culturel ; l'auteur se contente de présenter sous la forme de ğadwal des listes (avec quelques éléments biographiques) des hauts dignitaires de l'État ottoman. La tradition de l'idéal du prince n'est pas le cadre de pensée de ce savant, comme c'était le cas du lettré timouride, Mu'īn al-Dīn al-Națanzī.

La Bibliothèque nationale de France conserve un autre taqwìm de l'époque ottomane qui fut composé par un auteur anonyme ${ }^{93}$. Le texte, d'après les dernières informations datées qu'il renferme avant la conclusion, fut vraisemblablement rédigé au début du XVIII siècle. Il est, en quelque sorte, calqué sur le Taqwīm al-tawārīh de Kātib Čelebi ${ }^{94}$. L'auteur a recopié le Taqwìm de ce dernier pour les événements antérieurs à lui, mais le texte contient beaucoup plus d'informations et de commentaires, en particulier dans les marges. Ce Taqwim anonyme ne possède pas de titre, mais on trouve sur la couverture du manuscrit une marque de possession. Il appartenait à un nommé Meḥmed Emīn qui était, d'après le

89. Kātib Čelebi (1017-1067/1609-1657) est l'un des plus grands savants du XVII siècle dans l'Empire ottoman, voir O. S. GÖKYAY « Kātib Čelebi », EI², vol. IV, p. 791-792. Sur son Taqwīm al-tawārīh, voir le point bibliographique, la tradition manuscrite, les éditions et traductions in F. BABINGER, Die Geschichtsschreiber der Osmanen und ihre Werke, Leipzig, Otto Harrassowitz, 1927, p. 196-197. On trouvera également de nombreuses informations sur ce texte dans G. HAGEN, Ein osmanischer Geograph bei der Arbeit Entstehung und Gedankenwelt von Kātib Čelebis Ǧihānnümāa, Berlin, Klaus Schwartz Verlag, 2003.

90. G. HAGEN, Ein osmanischer Geograph, p. 59.

91. G. HAGEN, Ein osmanischer Geograph, p. 60.

92. G. HAGEN, Ein osmanischer Geograph, p. 247.

93. Le manuscrit est conservé à la BNF sous la cote Suppl. turc 1149.

94. Voir en annexe XIII, fig. 14, Kātib Čelebi, fol. 16r et fig. 15 fol. 6v du Suppl. turc 1149. 
nom de sa fonction (čuqadār-i enderūn), le page du sultan en charge de sa garde-robe. Il était par conséquent en contact direct avec lui et avait accès à sa "chambre privée". Comme beaucoup des personnels des services intérieurs du Palais, les čuqadār-i enderūn recevaient une éducation religieuse et lettrée par tout un personnel d'enseignants. On peut donc émettre l'hypothèse que Mehmed Emīn avait peut-être acheté ce manuscrit afin d'acquérir rapidement une culture historique qui pouvait lui être utile dans l'exercice de sa fonction auprès du sultan.

Ces deux auteurs se préoccupent essentiellement de l'histoire de l'islam et ne consacrent que peu de ğadwal aux rois de l'Iran; en tout cas, les tableaux qui leur sont dévolus sont réduits à leur plus simple expression. Les événements depuis le début de l'hégire sont classés par tranches de huit années dans Kātib Čelebi et de dix années chez l'auteur anonyme. Il n'est pas surprenant que la partie la plus riche en informations de ces deux taqwìm soit consacrée à l'histoire des Ottomans. Les auteurs présentent sous la forme de ğadwal la liste des grands personnages de l'État, ce qui nous informe sur la hiérarchie des fonctions dans l'Empire : sultans, grands vizirs, šayh̆-i islām ${ }^{95}$, quḍatt-i 'asākir, ḩwāğagān-i salāțīn-i 'Uțmanān, etc. On trouve des ğadwal consacrés aux quḍăt-i 'asākir de Constantinople et de chaque région de l'Empire, aux nāqib-i ešrāf, c'est-à-dire ceux qui étaient chargés de vérifier le nasab des personnes qui prétendaient descendre du Prophète. On trouve également des ğadwal mentionnant les "directeurs de conscience" des sultans (ḩwāğagān-i salātīn-i 'Uțmanān). Ces derniers ne détenaient pas une véritable fonction officielle, elle dépendait surtout de la personnalité du personnage en question et des liens qu'il entretenait avec le sultan à qui il prodiguait des conseils en particulier sur des questions religieuses. En réalité, certains de ces hwwāğagān ont détenu un pouvoir important puisqu'il leur arrivait d'intervenir dans la nomination des šayh al-islām. Ces chronographies présentent un très bon résumé de la hiérarchie des fonctions dans l'Empire ottoman, mais aucune information d'ordre culturel.

\section{CONCLUSION}

Manifestement, ces taqwīm historiques apparaissent essentiellement au XIV siècle et plus particulièrement dans les mondes persan et turc. Jusqu'à présent, je n'ai trouvé qu'un seul texte de ce type en arabe, anonyme et sans titre, dont l'écriture montre qu'il est aussi du XIV ${ }^{\mathrm{e}}$ siècle, mais il a pu être rédigé avant cette date ${ }^{96}$. Le manuscrit comporte environ 60 folios qui traitent des souverains musulmans du monde arabe. Dans des colonnes séparées, on trouve des informations sur chaque souverain : nom du père, de la mère, nom patronymique, laqab, année de naissance, date d'intronisation, date de la mort, longueur du règne, cause de la mort et lieu d'inhumation. Ces rubriques se rapprochent tout à fait de celles que Mu'īn al-Dīn al-Națanzī avait choisies, sauf que les qualités et les talents personnels des souverains ne sont pas mentionnés. Ce taqwìm historique fait suite à un ouvrage attribué à Abū l-Fidā', le Tibr al-maskūb. Ces deux textes semblent avoir été composés par le même

95. Dans l'Empire ottoman, la fonction de šayḩ al-islām est associée à celle de muftī de la capitale.

96. Il est conservé à Dār al-Kutūb au Caire sous la cote Ta'rīh 86m. 
auteur, mais le Tibr al-maskūb s'achève dix années après la mort d'Abū l-Fidā' ${ }^{97}$. L'attribution de cette histoire sous forme de tables n'est pas facile à résoudre. En tout cas, jusqu'à preuve du contraire, l'histoire sous la forme de ğadwal ne semble pas avoir connu un réel succès dans le monde arabe où l'écriture de l'histoire est restée beaucoup plus proche des modèles traditionnels qui ont vu le jour au début de l'islam.

D'après ce que l'on peut tirer des textes présentés ici, le but semble être d'ordre pédagogique : faciliter un accès rapide à des informations, même finalement dans le cas de Mu'īn al-Dīn al-Națanzī qui résume sous la forme d'un ğadwal à la fin du récit de l'histoire de chaque dynastie les faits historiques qui semblent marquants à ses yeux. Il cherche aussi à donner une image personnelle de chaque membre du lignage en mentionnant dans les ğadwal des informations d'ordre culturel et sur leur conduite personnelle qui ne figurent pas dans le texte. Nous avons pu constater que le choix des rubriques nous renseigne sur le milieu culturel des auteurs : lettrés en ce qui concerne les deux taqwìm persan; savants plus traditionnels pour les auteurs des textes rédigés en arabe et en milieu ottoman. Il est évident que les informations relatées dans les chronographies ottomanes ne peuvent servir au chercheur qui, lui, a recours aux détails qu'il peut trouver dans les "grandes chroniques". Il s'agit de manuels, faciles à consulter, pour obtenir le nom et des éléments biographiques sur un grand personnage de l'Empire.

Les ouvrages qui combinent généalogies et textes narratifs ont une grande importance pour mettre en lumière la manière dont évoluent les canons historiques. En effet, ils réinterprètent les événements en accord avec des modèles de filiation. Comme on l'a vu, le Šu'ab-i panğgānah de Rašīd al-Dīn et le Mu'izz al-ansāb déploient l'histoire sous la forme d'un principe de succession héréditaire dont l'un des buts est manifestement politique: inscrire les Timourides comme les héritiers et les successeurs légitimes de l'Empire mongol, l'autre plus culturel, préserver une image sociale de ces dynasties nomades de culture principalement orale.

Enfin, la question la plus délicate à résoudre est celle de l'origine ou des origines de cette présentation graphique de l'histoire. Les taqwim historiques ont surtout connu un développement relativement important, à partir du XIII-XIV siècle, dans une zone de contacts entre Turcs, Persans et chrétiens syriaques. Ces derniers avaient accès à l'arabe, au persan et au turc, comme en atteste le rôle qu'ils ont joué comme interprètes à l'époque mongole ${ }^{98}$. La culture syriaque a été un vecteur important de transmission culturelle entre mondes chrétien oriental et islamique. Il se pourrait que l'origine lointaine de ces taqwìm historiques islamiques soient les chroniques syriaques comme la Chronographie d'Élie de Nisibe qui combine la relation des événements année par année de manière résumée sous la forme de tables chronologiques en deux colonnes, l'une en syriaque et l'autre en arabe, dans la première partie du texte ${ }^{99}$ et qui rejette en seconde partie les calendriers des

97. Voir F. Rosenthal, A History of Muslim Historiography, p. 146, note 1.

98. Voir D. SINOR, «Interpreters in Medieval Inner Asia », Asian and African Studies, vol. 16 (1982), p. 293-320 et D. Aigle, « The Letters of Eljigidei, Hülegü and Abaqa: Mongol Overtures or Christian Ventriloquism? », Inner Asia 7/2 (2005), p. 143-162.

99. Voir en annexe XIV, fig. 16, Suppl. turc 1149, les événements du début de l'islam et fig. 17, la reproduction du fol. 29v de l'unique manuscrit de cette Chronographie qui est conservé au British Museum, Add. 7197, reproduite in 
différents peuples. Les taqwìm ottomans ressemblent beaucoup dans leur présentation à la Chronographie d'Élie de Nisibe, mais pour les historiens ottomans, les canons chronologiques syriaques, trop compliqués pour le lectorat auquel ce type de texte était destiné, sont réduits, comme on l'a vu, à leur portion congrue.

En tout cas, dans l'état actuel de nos connaissances, il est difficile de trancher sur l'origine de cette présentation de l'histoire sous forme de ğadwal. En effet, on peut également imaginer que ce sont les zĭg qui sont à la source de cette présentation graphique de l'histoire ou encore les "carrés magiques". Mais, si tel était le cas, on aurait utilisé plus tôt les taqwìm dans l'historiographie ou dans la littérature "para-historique". On a vu que des scientifiques musulmans (en astronomie et en médecine) ont cherché à simplifier assez tôt des ouvrages traduits du grec en arabe dans un but pédagogique. Cependant, d'après les recherches que j'ai pu faire jusqu'à présent sur cette présentation de l'histoire sous forme graphique, ces textes semblent bien apparaître, même si l'on dispose de quelques exemples antérieurs, au XIII ${ }^{\mathrm{e}}-\mathrm{XIV}^{\mathrm{e}}$ siècle, qui a vu un grand essor de la littérature historique islamique. Les taqwìm historiques semblent bien appartenir plutôt à la tradition turco-persane qu'à la tradition arabe. La question de l'origine ou des origines de ces taqwim historiques reste pour l'instant ouverte. Elle ne pourra progresser que lorsque seront effectuées de nouvelles investigations dans les fonds des bibliothèques, notamment dans les bibliothèques d'Istanbul qui conservent des ouvrages historiques de ce type, mais auxquels je n'ai pas encore eu accès. Cependant, les textes que j'ai déjà identifiés sont rédigés en persan ou en turc, ce qui pourrait confirmer les hypothèses émises ci-dessus.

D. WeLTECKE, Die «Beschreibung der Zeiten» von Mōr Michael dem Grossen (1129-1199), p. 190. 


\section{BIBLIOGRAPHIE}

\section{Sources manuscrites}

IBN AL-FuwAṬī (Kamāl al-Dīn Abū l-Faḍl ‘Abd al-Razzāq al-Šaybānī al-Hanbalī), Talhịṣ mağma' al-ādāa fì mu'ğam al-alqāb, manuscrit conservé à la Maktabat al-Asad à Damas sous la cote Ta'rīh 267 du fonds Zahiriyya.

IBN ĞAZLA,

Taqwìm al-abdān, manuscrit à Bagdad.

KĀTIB ČELEBI, Taqwīm al-tawārīh, manuscrit conservé à la BNF à Paris sous la cote Suppl. persan 1739.

Muğmal al-tawārīh wa l-qișaș, Manuscrit conservé à la BNF à Paris, sous la cote Persan 62.

Mu'IZZ, Al-ansāb, Manuscrit conservé à la BNF à Paris sous la cote Suppl. persan 73.

AL-NAṬANZī, Mu'īn al-Dīn, Muntahab al-tawārīh, manuscrit conservé à la BNF à Paris sous la cote Suppl. persan 1651.

Nisibie, Élie (de),

Chronographie, manuscrit conservé à la British Library à Londres sous la cote Add. 7197.

RAŠĩD AL-DīN,

Ğāmi' al-tawārīh, manuscrit conservé à la BNF à Paris sous la cote Suppl. persan 1113. Šu'ab-i Panğgānah, manuscrit conservé à Topkapi Saray.

AL-SABZAWĀR̄i, Kamāl al-Dīn Husayn b. 'Alī,

Mahzan al-inš $\bar{a}$ ', manuscrit conservé à la BNF à Paris sous la cote Persan 73.

Șahîfa-i Šāhī, manuscrit conservé à la BNF à Paris sous la cote Suppl. persan 1059.

Taqwīm historique, sans titre manuscrit conservé à l'Institut français de recherche en Iran sous la cote Ms 18.

Taqwim historique ottoman, sans titre manuscrit conservé à la BNF à Paris sous la cote Suppl. turc 1149.

Ṭūsī (AL-), Nașīr al-Dīn, Tahrīir al-maǧisțī, manuscrit conservé à la BNF à Paris sous la cote Arabe 2485.

\section{Sources imprimées}

Chronique de Michel le Syrien

1899-1910 Patriarche jacobite d'Antioche (1166-1199), 4 vol., J.-B. Chabot (éd. et trad. du fac-similé), Paris, Ernest Leroux [Réimpression anastatique de l'édition de Paris, Bruxelles, Culture et civilisation, 1963].

La chronographie d’Élié Bar Šinaya.

1910 Métropolitain de Nisibe. Traduite pour la première fois d'après le manuscrit Add. 7197 du Musée britannique par L.-J. Delaporte, Paris, Honoré Champion. 
Chronologie orientalischer Völker von Albêrûni,

1923 C. E. Sachau (éd.), Leipzig, Otto Harrassowitz [1 $1^{\mathrm{er}}$ éd. 1878].

Eliae metropolitae Nisibeni.

1909-1910 Opus chronologicum, E. W. Brooks et J.- B. Chabot (éd.), 2 vol., Paris (Corpus scriptorum christianorum orientalium, 62-63. Scriptores Syr. 21-24).

Ġāzān-nāma, M. Tadbbūrī (éd.), Téhéran, 1381š.

Géographie d'Aboulféda.

1840 Texte arabe publié d'après les manuscrits de Paris et de Leyde aux frais de la Société asiatique par M. Reinaud et M. le baron Mac Guckin de Slane, Paris, Imprimerie royale.

Muğmal al-tawārīhn wa l-qișaṣ

2001 Édition en fac-similé du manuscrit copié en 751/1350, conservé à la Staatsbibliothek de Berlin, par I. Afshar et M. Omidsalar, Téhéran (Society for Promotion of Persian Culture, Indiana).

AL-NAȚANZī, Mu'īn al-Dīn,

1957 Muntahab al-tawārīhn-i Mu`īn̄̄, J. Aubin (éd), Téhéran, librairie Khayyam.

Rashīd al-Dīn Faẓl AllāH al-Hamadānī,

2000 History of China and Cathay. Being a fragment of Jāmit at-Tawārīkh, Wang Yidan (éd.), Téhéran, Iran University Press.

2005 Jāmi' al-Tavārīkh (The History of Afranj, Popes and Caesars), M. Rushan (éd.), Téhéran, Mīrāt-i Maktūb.

2005 Jāmi' al-Tavārīkh (The History of India, Indus and Cashmere), M. Rushan (éd.), Téhéran, Mīrāt-i Maktūb.

2005 Jāmi al-Tavārīkh (History of Ughūz), M. Rushan (éd.), Téhéran, Mīrāte-i Maktūb.

Le Taqwìm al-Ṣiḥha (Tacuini Sanitatis) d'Ibn Buțlān: un traité médical du XIe siècle, histoire du texte, édition critique, traduction, commentaire par H. Elkhadem, Louvain, Peeters, 1990.

YūSUF KHĀșS HĀJIB,

1983 Wisdom of Royal Glory (Kutadgu Bilig). A Turko-Islamic Mirror for Princes, translated, with an Introduction and Notes by R. Dankoff, Chicago/Londres, The University of Chicago.

\section{Études}

À l'ombre d'Avicenne. La médecine au temps des califes.

1996 Exposition présentée du 18 novembre 1996 au 2 mars 1997, Paris/Gand, IMA et SnoeckDucaju.

Aigle, D.,

2005 "The Letters of Eljigidei, Hülegü and Abaqa: Mongol Overtures or Christian Ventriloquism? », Inner Asia, 7/2, p. 143-162.

2007 «La conception du pouvoir en islam. Miroirs des princes persans et théories sunnites (XI-XIVe siècles) », Perspectives médiévales, vol. 31, p. 17-44.

Allsen, Th. T.,

Culture and Conquest in Mongol Eurasia, Cambridge, Cambridge University Press.

ARAT, R. R.,

1936-1939 «Fatih Sultan Mehmed'in yarlığı», Tükiyat Mecmuasi, vol. 6, p. 255-322 + reproduction du document en fac-similé, $20 \mathrm{p}$.

Aubin, J.,

1957 «Introduction », in Extraits du Muntakhab al-tavarikh-i Mu'ini (Anonyme d'Iskandar), J. Aubin (éd.), Téhéran, librairie Khayyam, p. 1-8. 
BABINGER, F., 1927

BLochEt, É,

1933

1934

BоIот, D. J.

BuelL, P. D.,

Colin, G. S.,

(Van), B.

$$
\text { « Ta'rīkh », EI², vol. X, p. 283-290. }
$$

DeBIÉ, M., 2006

DeWeEse, D., 2006

DiнHUDĀ, 'A., s.d. "L'héritage de la chronique d'Eusèbe dans l'historiographie syriaque », Journal of the Canadian Society for Syriac Studies, vol. 6, p. 18-26.

Historical Dictionary of the Mongol World Empire, Lanham, Maryland et Oxford, The Scarecrow Press.

$$
\text { « Abdjad », EI } I^{2} \text {, vol. I, p. } 100 .
$$

«Cultural transmission and exchange in the Mongol Empire: Notes from the biographical Dictionnary of Ibn al-Fuwațī », in Beyond the Legacy of Genghis Khan, L. Komaroff (éd.), Leyde, Brill, p. 11-29.

Fouchécour (de), Ch.-H.,

1986 Moralia. Les notions morales dans la littérature persane $d u 3^{e} / 9^{e}$ au $7^{e} / 13^{e}$ siècle, Paris, éditions Recherche sur les civilisations (Synthèse $n^{\circ} 23$ ).

GEARY, P. J.,

1996 La mémoire et l'oubli à la fin du premier millénaire, traduit de l'anglais par Jean-Pierre Ricard, Paris, Aubier.

GiвB, H. A. R.,

$$
\text { « Abū l-Fidā’», EI², vol. I, p. } 122 .
$$

GÖKYAY, O. S.,

« Kātib Čelebi », EI², vol. IV, p. 791-792.

HAGEN, G., 2003

Ein osmanischer Geograph bei der Arbeit Entstehung und Gedankenwelt von Kātib Čelebis Ğihānnümā, Berlin, Klaus Schwartz Verlag.

HofELICH, M.,

$$
\text { « Takwīm », EI², vol. X, p. 136-138. }
$$

Islamic aera studies with information systems, O. Atsuyuki (éd), Londres, Routledge Curzon, 2004.

\section{KenNEDY, E. S. et M. H.,}

1987 Geographical coordinates of localities from Islamic sources, Francfort, Institüt für Geschischite der Arabisch-Islamwissenschaft. 
Khaleghi-Motlagh, Dj. «Adab», EIr, vol. I, p. 433-435.

KING, D. A.,

1975 «On the Astronomical Tables of the Islamic Middle Ages », in Colloquia Copernicana III, p. 37-56.

King, D. A. et SAmsó, J.,

« Zīdj », EI², vol. XI, p. 537-550.

KöPRÜLÜ, M. F.,

2005 « Osmanlī », partie II, « La littérature », EI², vol. VIII, p. 214-223.

L'âge d'or des sciences arabes. Exposition présentée à l'Institut du monde arabe, Paris 25 octobre 2005-19 mars 2006, Arles, Actes Sud/IMA.

MANZ, B. Forbes,

1988 «Tamerlane and the Symbolism of Sovereignty », Iranian Studies, vol. 21/1-2, p. 105122.

1989 The Rise and Rule of Tamerlane, Cambridge, Cambridge University Press.

2000 "Mongol History rewritten and relived», in Figures mythiques des mondes musulmans, D. Aigle (dir.), Revue des mondes musulmans et de la Méditerranée, vol. 89-90, p. 129-149.

2002 "Tamerlane's Career an its Uses », Journal of World History, vol. 13/1, p. 125.

Melvilie, Ch.,

1994 "The Chinese Uighur Animal Calendar in Persian Historiography of the Mongol Period », Iran, vol. 32, p. 83-98.

MERCIER, R.,

1984 "The Greek 'Persian Syntaxis' and the Zij-i Ikhānī », Archives internationales d'histoire des sciences, vol. 34, p. 33-60.

PINGREE, D.,

1962

« Historical Horoscopes », Journal of the American Oriental Society, vol. 82, p. 487-502.

1977

Preceptum Canonis Ptolemei, Louvain-la-Neuve, Bruylant-Academia.

QuinN, S. A.,

« The Mu'izz al-Ansāb and Shu'ab-i Panjgānah as Sources for the Chaghatayid Period of History: A Comparative Analysis », Central Asiatic Journal, vol. 33/3-4, p. 229- 253.

RICHARD, F.,

1989 Catalogue des manuscrits persans I. Ancien fonds, Paris, Bibliothèque nationale.

RIEU, C.,

1895 Supplement to the Catalogue of the Persian MSS in the British Museum, Londres .

Rosenthal, F.,

1968 A History of Muslim Historiography, Leyde, Brill.

ScHACHT, J.,

« Ibn Buțlān », EI², vol. III, p. 763-764. 
SINOR, D.,

1982

«Interpreters in Medieval Inner Asia », Asian and African Studies, vol. 16, p. 293- 320.

2007

"Some Observations on Women in Early and Medieval Inner Asian History », in The Role of Women in the Altaic World. Permanent International Altaistic Conference, 44th Meeting, Walbergberg, 26-31 August 2001, V. Veit (éd.), Wiesbaden, Harrassowitz Verlag, p. 261268.

SOUDOVAR, A.,

1996 "The saga of Abu-Sa'id Bahādor Khān. The Abu-Sa'id nāmé », in The court of the Il-khans, J. Raby et T. Fitzherbert (éd.), Oxford, Oxford University Press, p. 95-218.

2006 "The Mongol Legacy of Persian Farmāns », in L. Komaroff (éd.), Beyond the Legacy of Genghis Khan, Leyde, Brill, p. 407-421.

SPIEGEL, G. M.,

The Past as Text. The Theory and Practice of Medieval Historiography, Baltimore et Londres, The Johns Hopkins University Press.

1997 "Genealogy. Form and Function in Medieval Historiography », in The Past as Text. The Theory and Practice of Medieval Historiography, Baltimore et Londres, The Johns Hopkins University Press, p. 99-110, notes, p. 242-245.

1997 «Social Change and Literary Language. The textualization of Past in ThirteenthCentury Old French Historiography ", in The Past as Text. The Theory and Practice of Medieval Historiography, Baltimore et Londres, The Johns Hopkins University Press, p. 178-194, notes, p. 271-280.

Sublet, J.,

1998 «Dans l'Islam médiéval, nom en expansion, nom à l'étroit : L'exemple d'Ibn alFuwațī », in L'écriture du nom propre, textes réunis et présentés par A.-M. Christin, Paris, L'Harmattan, p. 117-134.

THACKSTON, W. M.,

1989 "Anonymous Synoptic account of the House of Timur ", in A Century for Princes. Sources on Timurid History and Art. Selected and Translated by W. M. Thackston, Cambridge Massachusetts, The Aga Khan Program for Islamic Architecture, p. 237- 246.

Troupeau, G., 1993 Bulletin critique des Annales islamologiques, vol. 10, p. 193-195.

VERNET, J.,

WELTECKe, D., 2003

« Ibn Djazla », EI², vol. III, p. 776-777.

Die «Beschreibung der Zeiten» von Mōr Michael dem Grossen (1129-1199). Eine Studie zu ihrem historischen und historiographie geschichtlichen Kontext, Louvain, Peeters (Corpus scriptorum christianorum orientalium, 594. Subsidia, t. 110). 
Annexe I

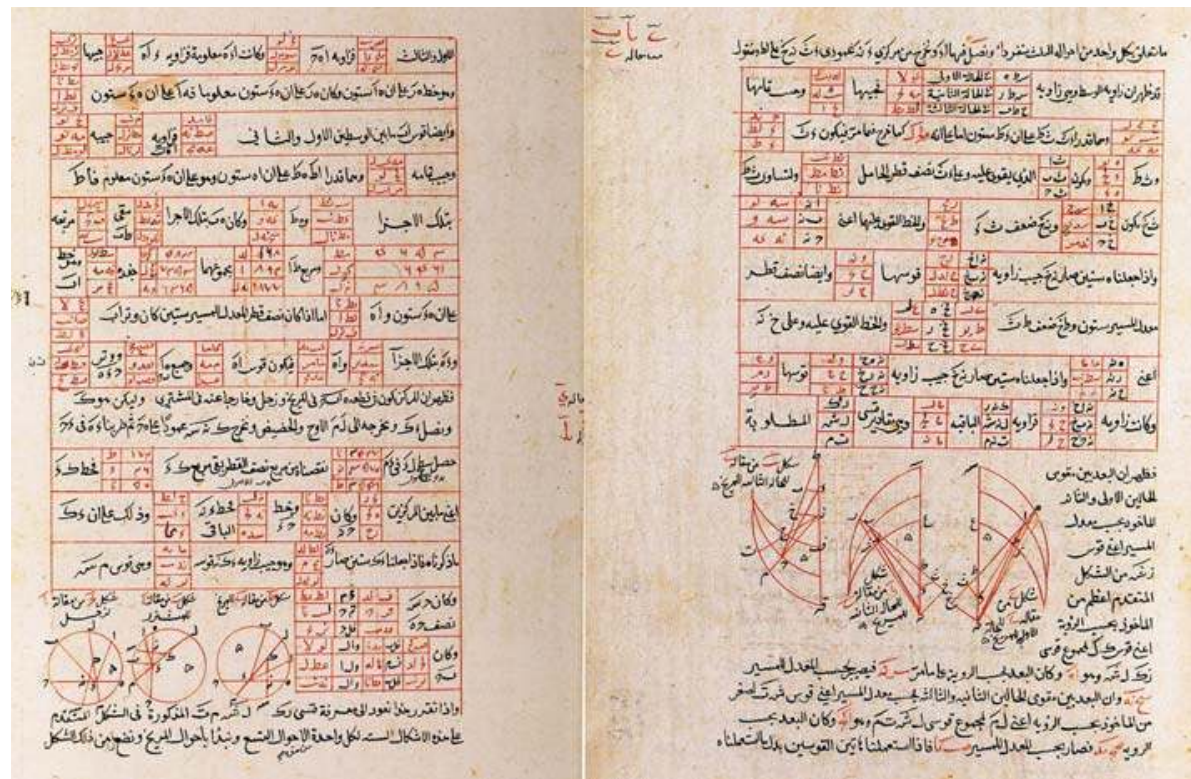

Fig. 1 : Nașīr al-Dīn al-Ṭūsī, Taḥrīr al-maǧistī, BNF Arabe 2485 fol. 83v-84r.

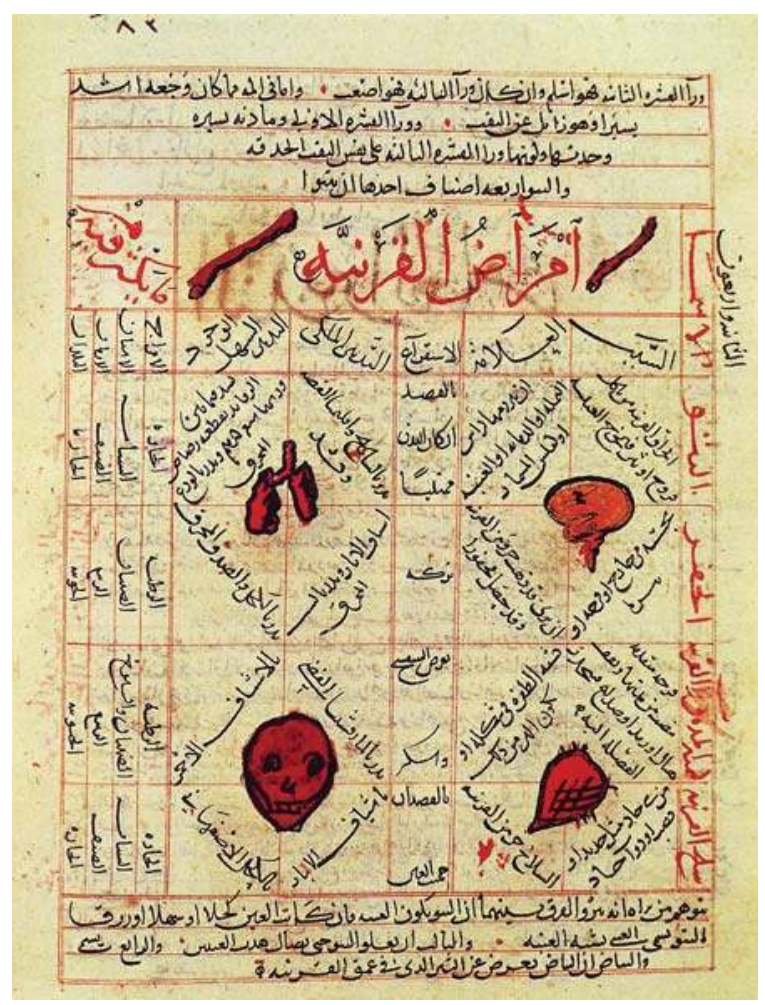

Fig. 2 : Ibn Ğazla, Taqwīm al-abdān, manuscrit copié au XV's. à Bagdad.

Reproduit in F. Micheau, Les Pays d'Islam VII - XVI siècle, Paris, La Documentation française, nº 8007, 1999, p. 40. 
Annexe II

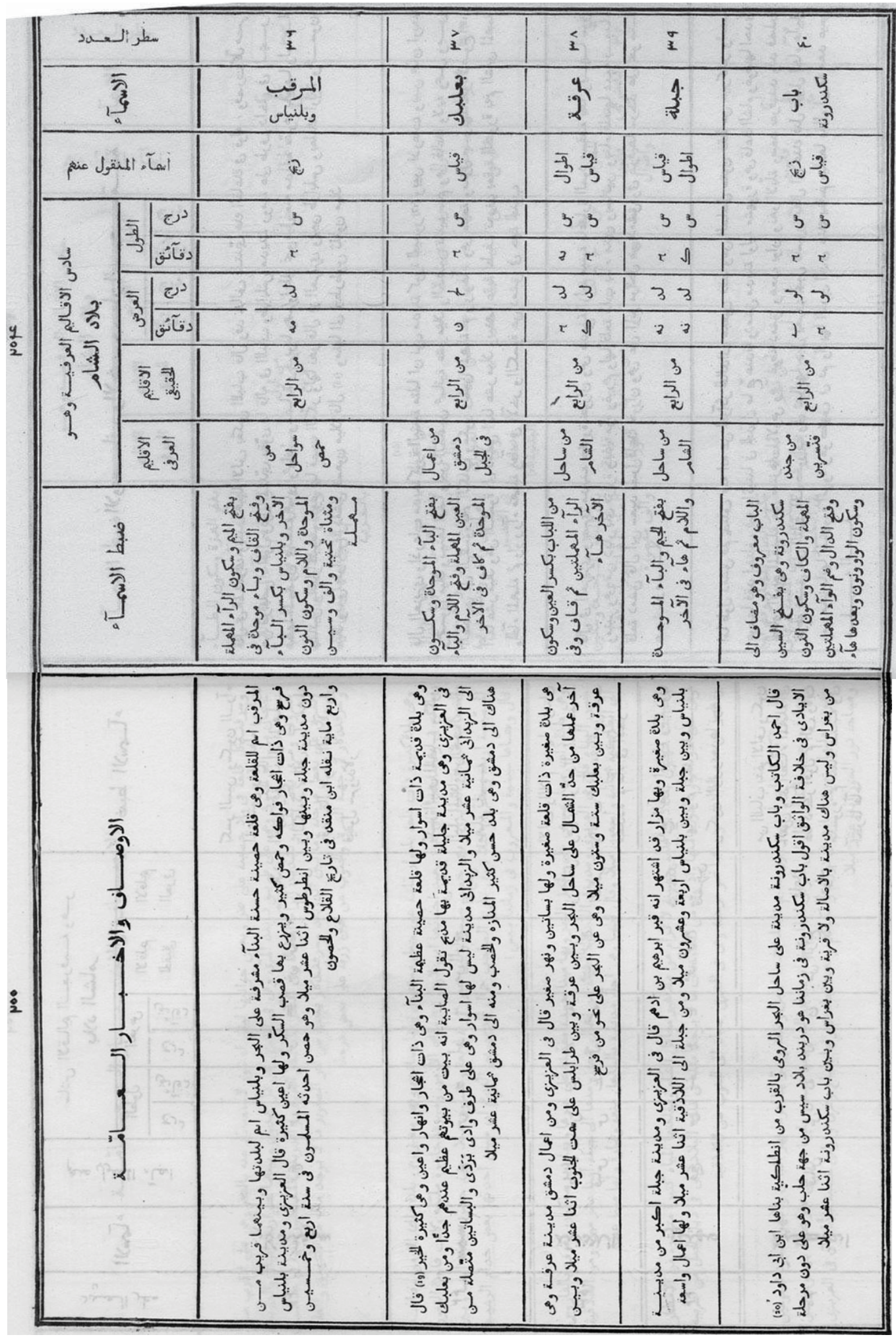


Annexe III

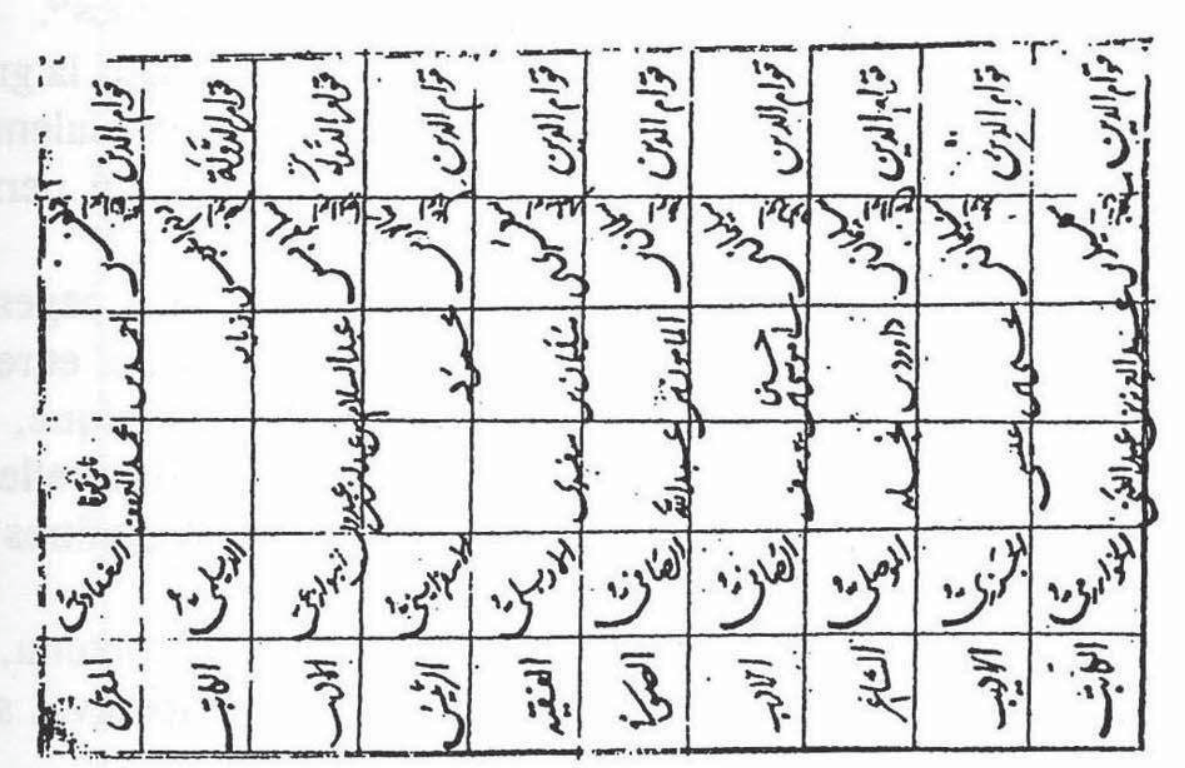

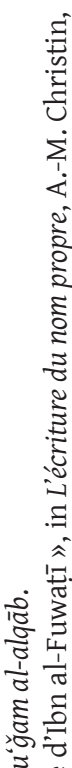
론 을 教 is 홍

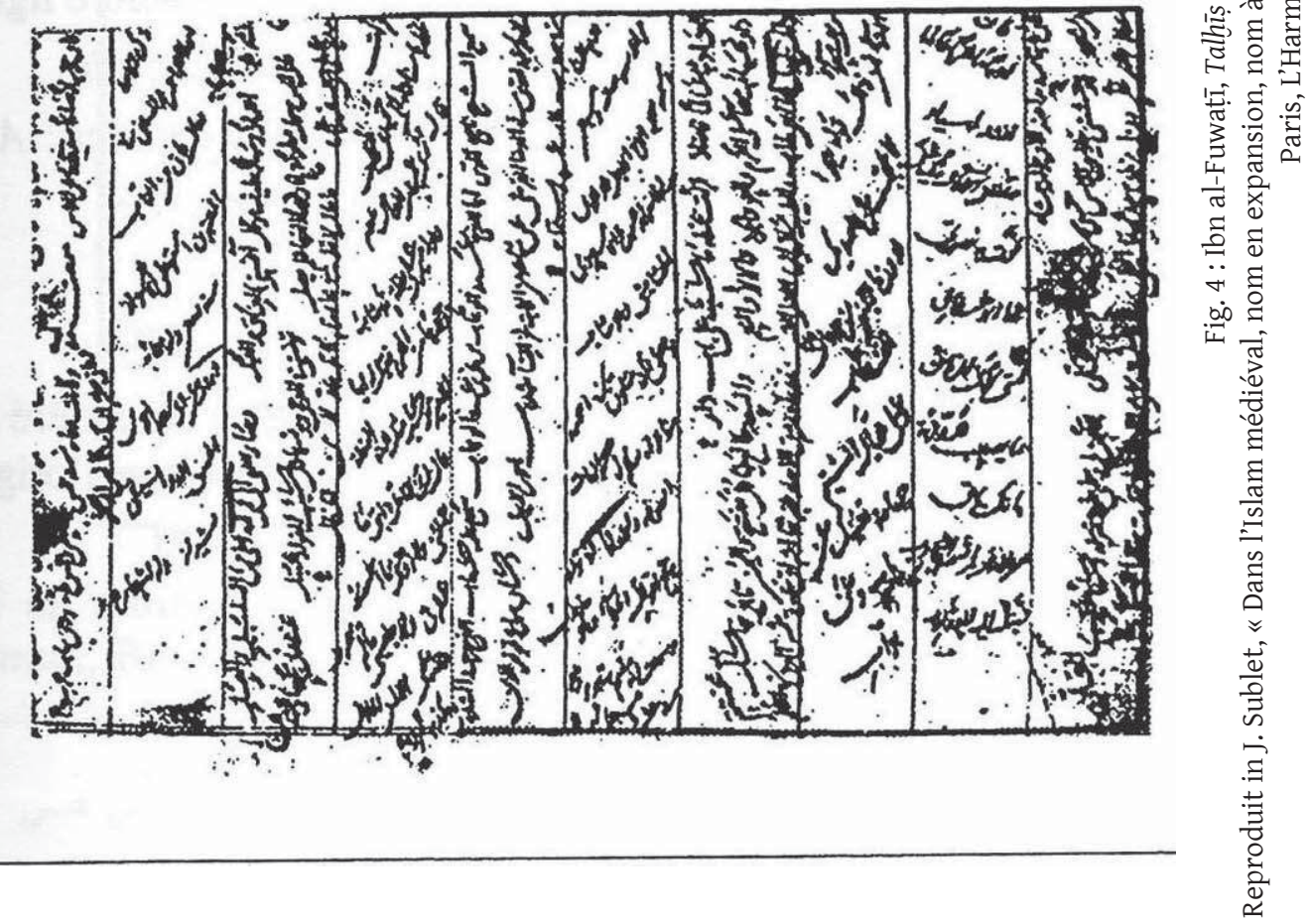


Annexe IV

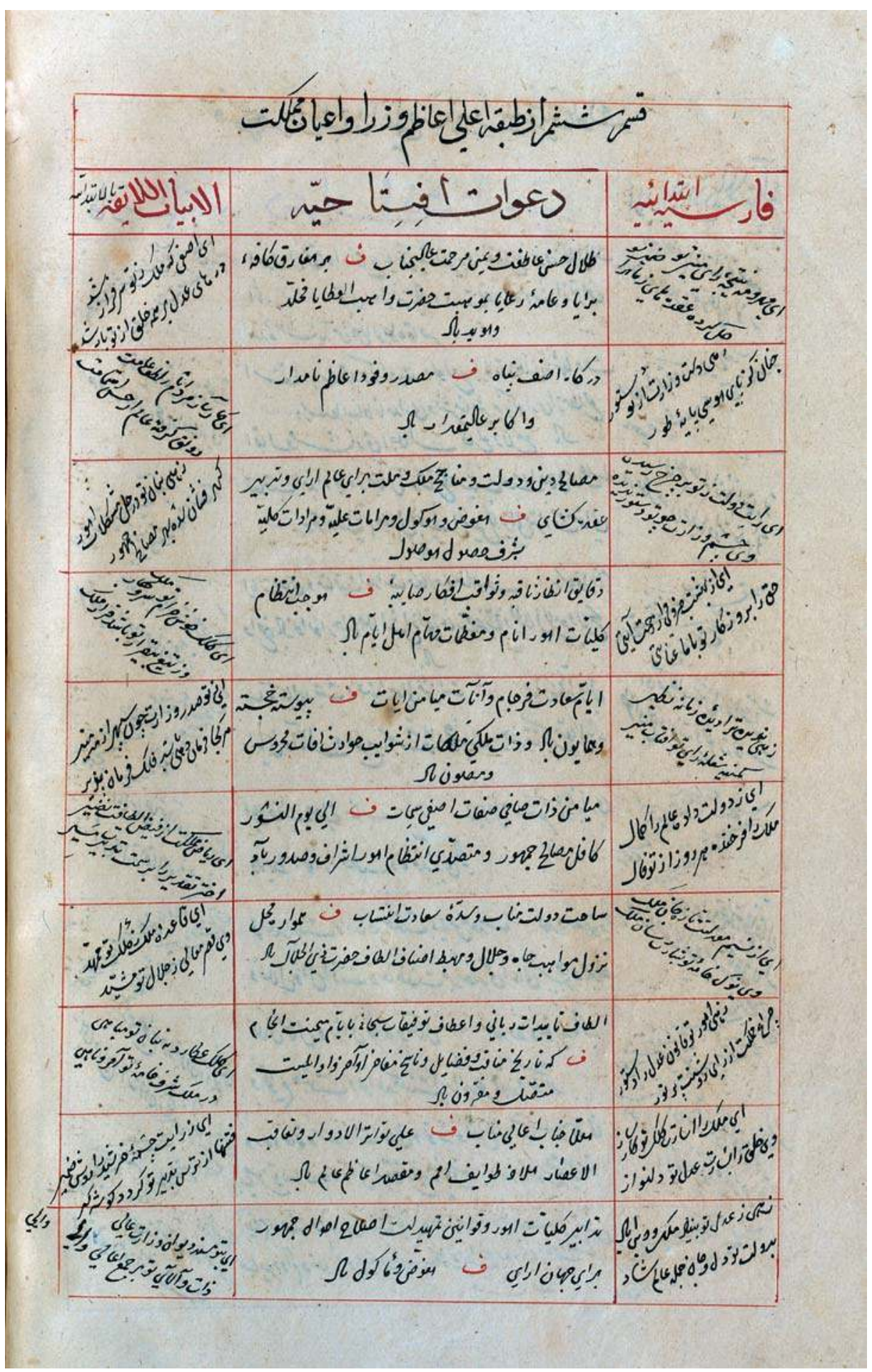

Fig. 5 : Kamāl al-Dīn Ḥusayn, Mahzzan al-inšă’, BNF Persan 73, fol. 6v. 
Annexe V

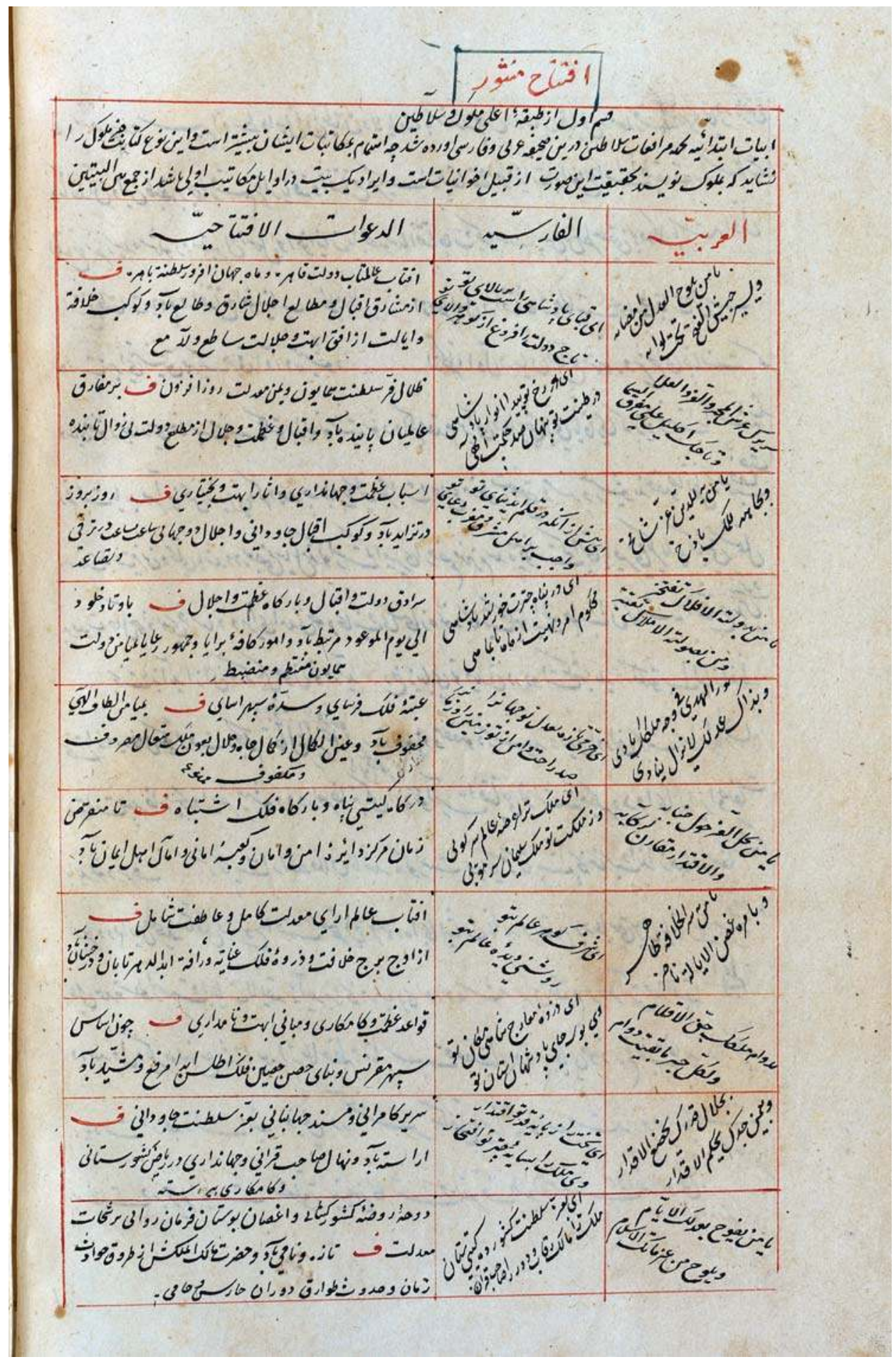

Fig. 6 : Kamāl al-Dīn Ḥusayn, Mahzan al-inšă', BNF Persan 73, fol. 7v. 


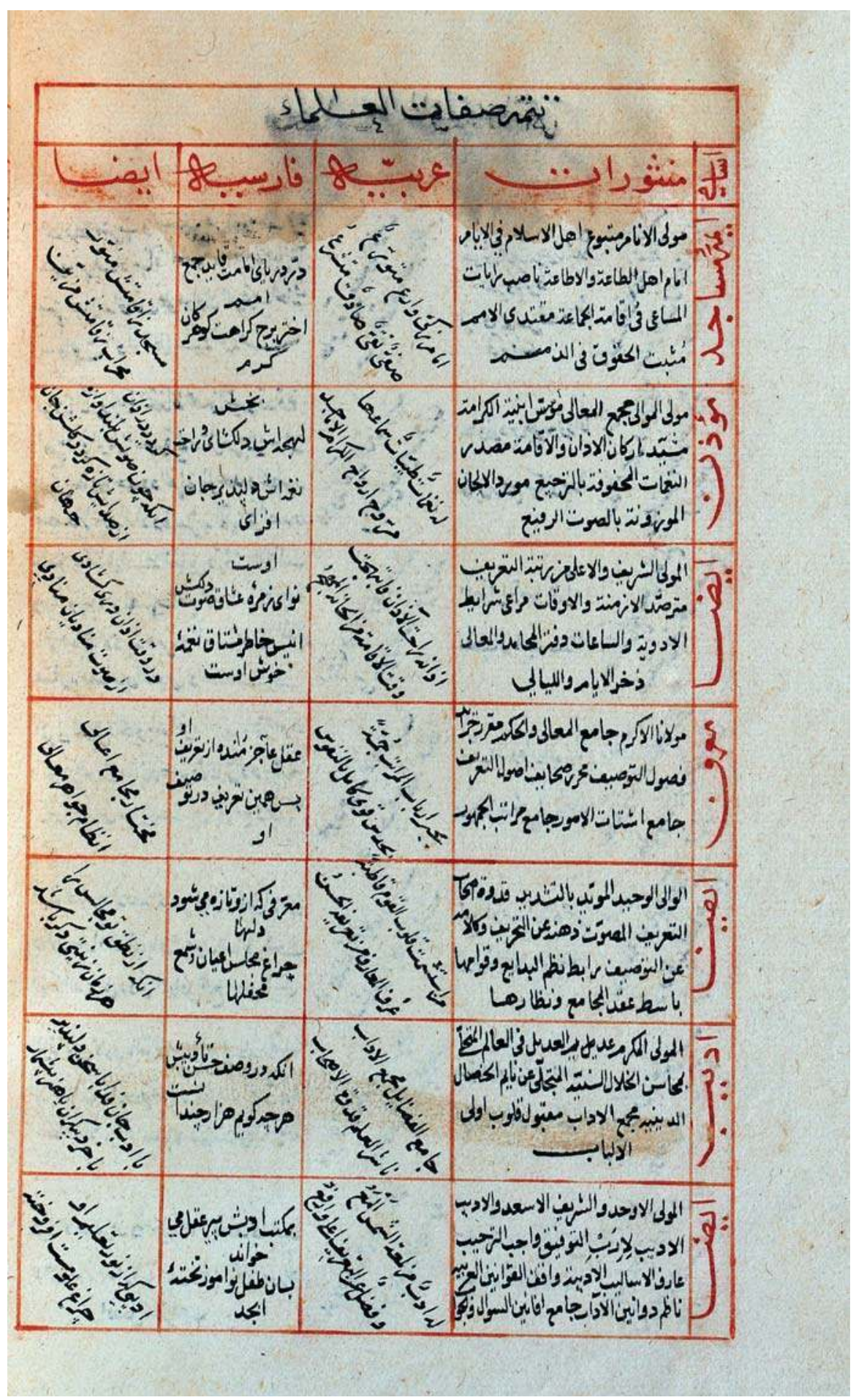

Fig. 7 : Kamāl al-Dīn Ḥusayn, Șahīfa-i $\check{S} a ̄ h \bar{l}$, BNF Suppl. persan 467, fol. 44v. 


\section{Annexe VII}

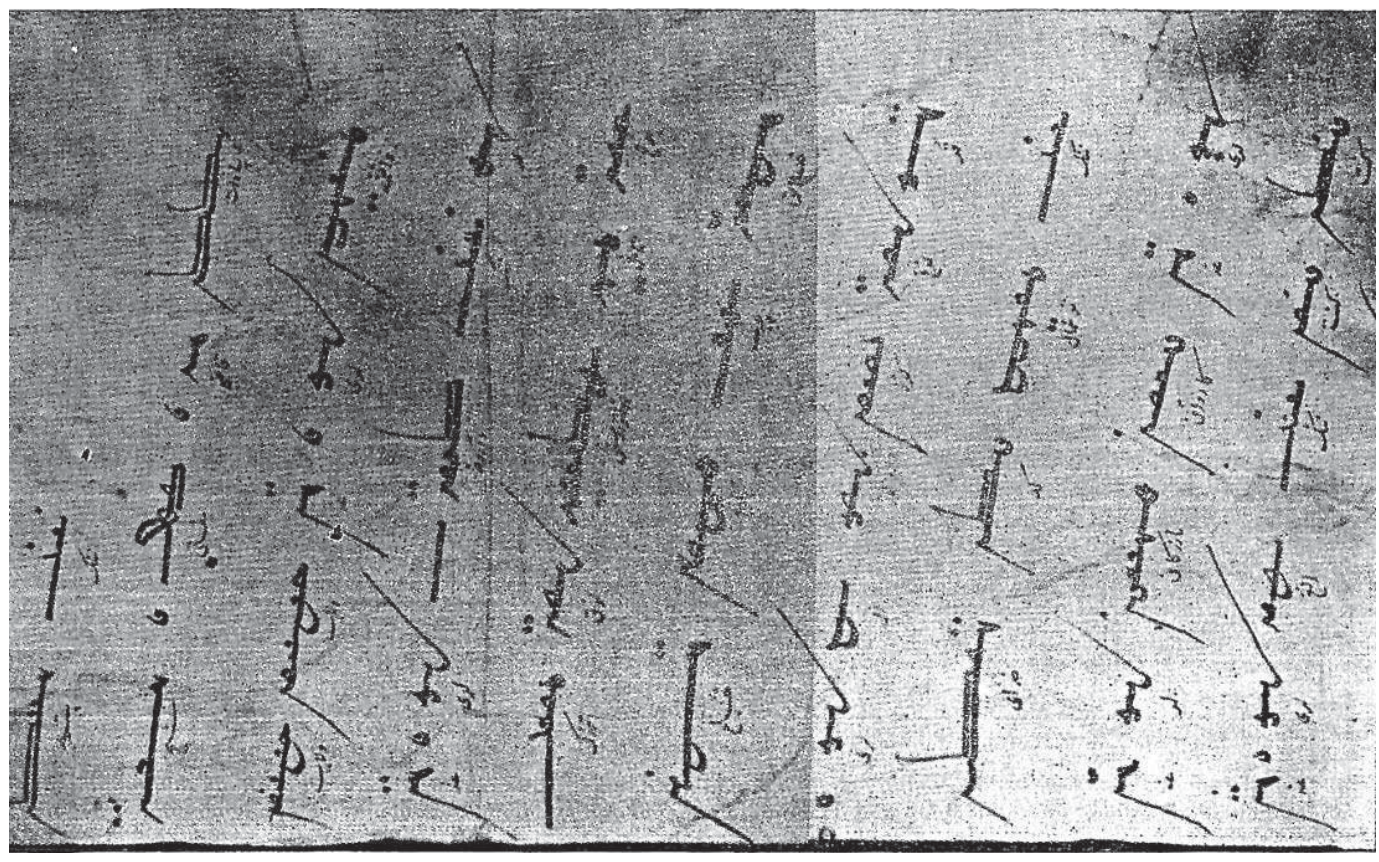



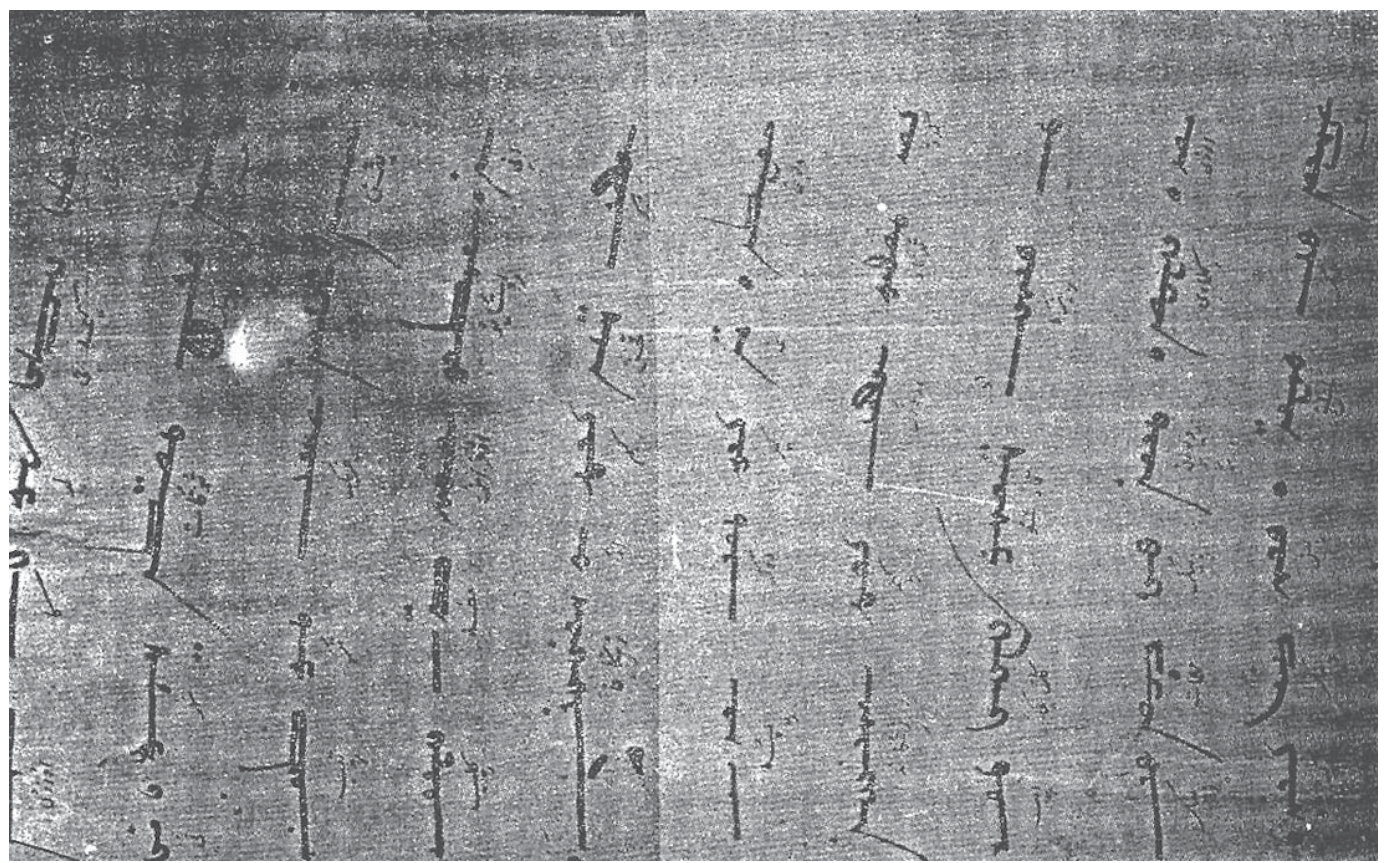

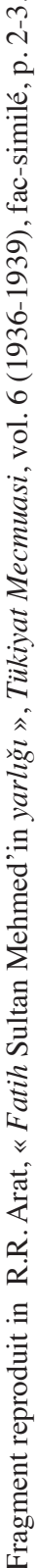




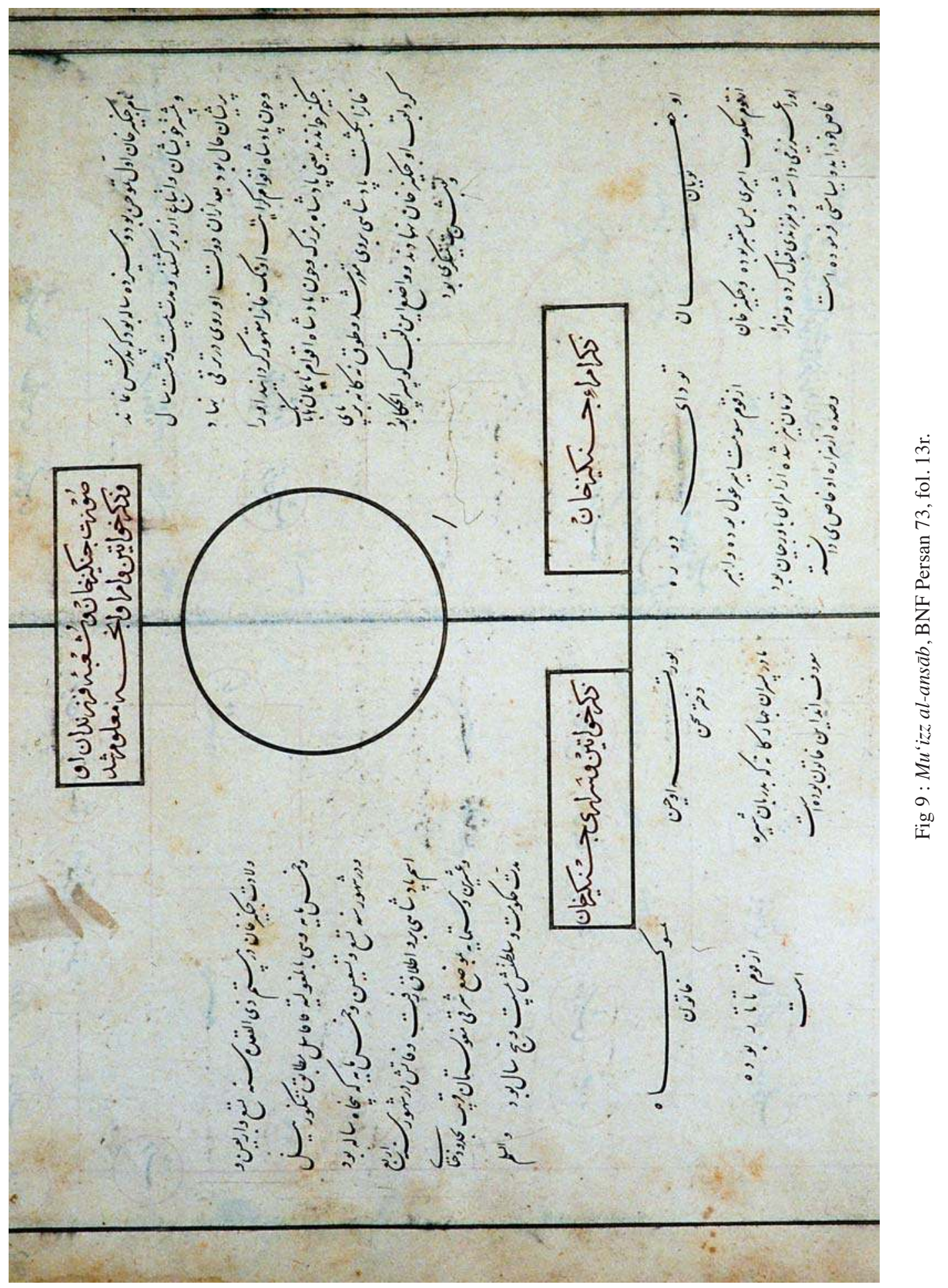




\section{Annexe IX}

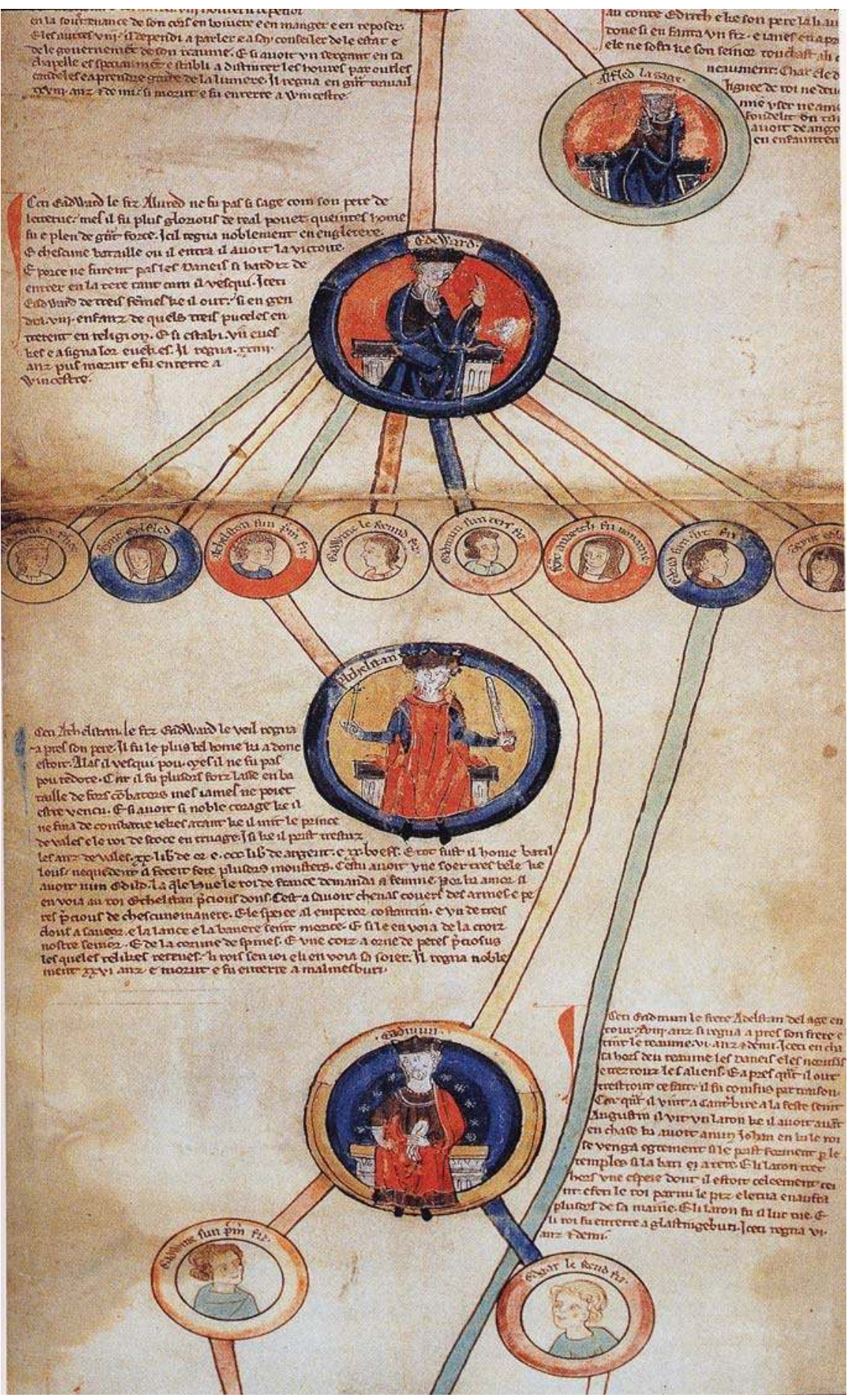

Fig. 10 : Chronique anglaise anonyme.

Fragment reproduit in D. Weltecke, Die «Beschreibung der Zeiten» von Mōr Michael dem Grossen (1129-1199), p. 187. 
Annexe X

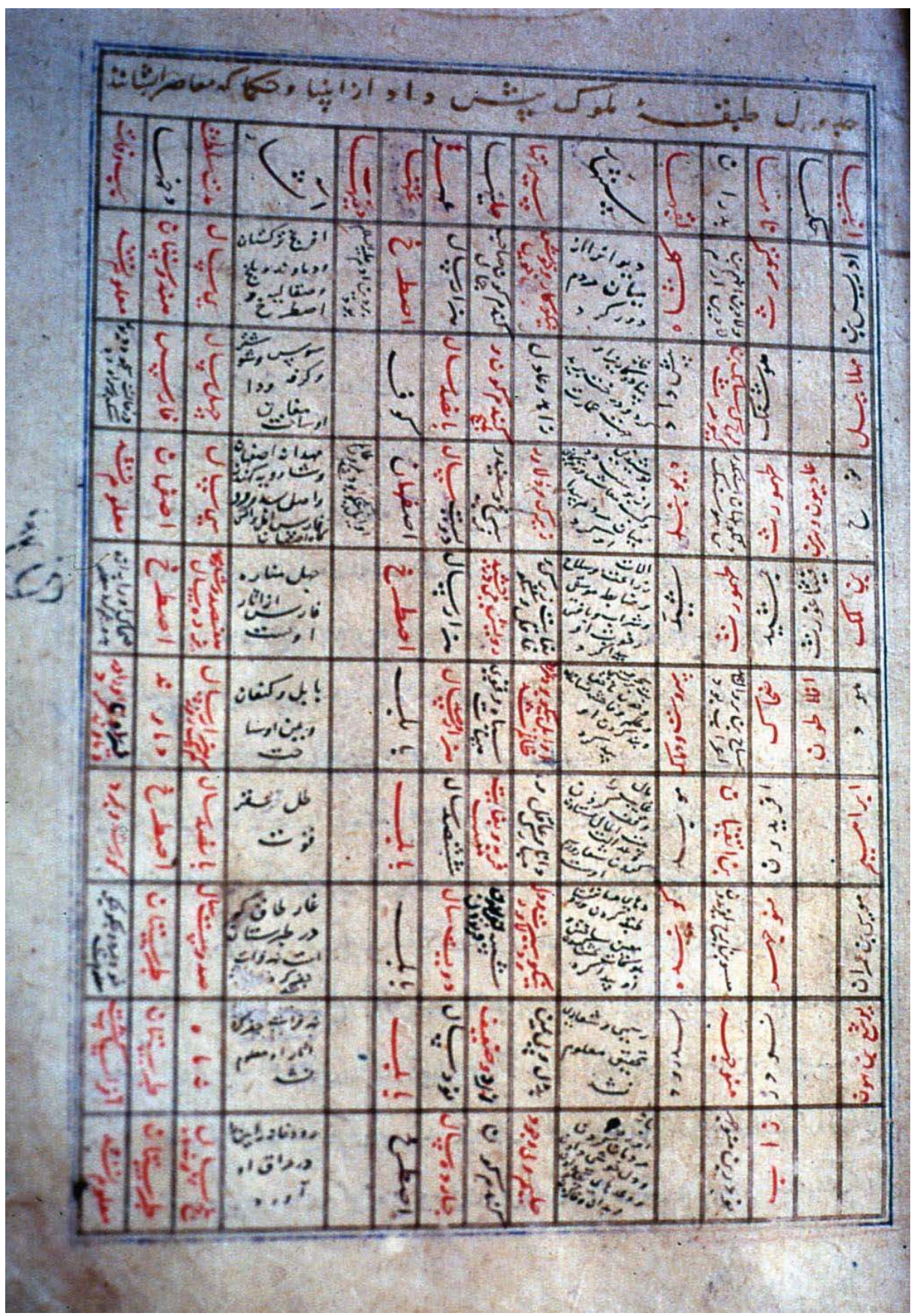

Fig. 11 : Mu'īn al-Dīn al-Națanzī, Muntahab al-tawārīh . BNF Suppl. persan 1651, fol. 12. 


\section{Annexe XI}

Ğadwal de Malik Ašraf Kučik et de ses descendants

\begin{tabular}{|c|c|c|c|}
\hline laqab & Kučik & Malik & Ǧamāl al-Dīn \\
\hline noms & Șayh Hasan & Ašraf & Pīr Hụsayn \\
\hline pères & Čūpān Ǧalā'ir & Tīmūr-Tāš b. Čūpān & Maḥmūd b. Čūpān \\
\hline \multicolumn{4}{|l|}{ mères } \\
\hline enfants & un fils & six fils & un fils \\
\hline hilyat-hā & teint mat & peau sombre & teint mat \\
\hline$p \bar{\imath} \check{s} \bar{a}-h \bar{a}$ & & $\begin{array}{l}\text { il écrivait son propre } \\
\text { sceau de sa main en } \\
\text { écriture turque }\end{array}$ & $\begin{array}{c}\text { il s'amusait dans } \\
\text { le bazar avec des } \\
\text { chaussures }\end{array}$ \\
\hline sīrat-hā & il buvait beaucoup & il était tyrannique & $\begin{array}{l}\text { il menait une vie } \\
\text { dissolue }\end{array}$ \\
\hline hunar-hā & il était très preux & $\begin{array}{l}\text { il était cultivé et } \\
\text { écrivait en écriture } \\
\text { turque }\end{array}$ & il n'avait pas de culture \\
\hline grands de l'époque & $\begin{array}{c}\text { Šayh Nūr al-Dīn } \\
\text { ‘Abd al-Raḥmān et } \\
\text { Šayḩ Șadr al-Dīn al- } \\
\text { Ardabīlī }\end{array}$ & $\begin{array}{c}\text { Šayh Nūr al-Dīn } \\
\text { ‘Abd al-Raḥmān et } \\
\text { Šayh Șadr al-Dīn al- } \\
\text { Ardabīlī }\end{array}$ & $\begin{array}{c}\text { Mawlānā A'žam Ğamāl } \\
\text { al-Dīn al-Fālī }\end{array}$ \\
\hline sceaux & Șayh Ḥasan b. Čūpān & & Pīr Hụsayn \\
\hline ministres & & & Hāăğğì Qawwām Buzurg \\
\hline grands émirs & Ašraf & Yāg̀īi-Bāstī & Muhammad-i Muzaaffar \\
\hline œuvres & une madrasa à Tabrīz & $\begin{array}{c}\text { il a construit un } \\
\text { château à la cour de } \\
\text { Tabrīz }\end{array}$ & \\
\hline date d'intronisation & 736 & 738 & 739 \\
\hline durée du règne & deux ans & sept ans & 5 ans \\
\hline date de la mort & 738 & 745 & 745 \\
\hline cause de la mort & $\begin{array}{c}\text { sa femme lui tordit les } \\
\text { testicules jusqu'à ce } \\
\text { qu'il meure }\end{array}$ & il fut tué & il fut tué \\
\hline lieu de sépulture & Tabrīz & Tabrīz & Marāga \\
\hline
\end{tabular}

Fig. 12 : Traduction du fol. 329v : Ğadwal de Malik Ašraf Kučik et de ses descendants. 
Annexe XII

\begin{tabular}{|c|c|c|c|}
\hline $\begin{array}{ll}\text { - juste ('ādil) } & \text { JUSTICE } \\
\end{array}$ & $\begin{array}{l}\mathbf{1 4} \\
10\end{array}$ & $\begin{array}{r}\text { INJUSTICE } \\
\text { - injuste ('ālim) }\end{array}$ & $\begin{array}{l}3 \\
3\end{array}$ \\
\hline - contentement $\left(q a n \bar{a}^{\prime} a\right)$ & 1 & & \\
\hline - actions en faveur de l'islam & 3 & & \\
\hline $\begin{array}{l}\text { SAVOIRS ET TALENTS } \\
- \text { savant }(d \bar{a} n \bar{a})\end{array}$ & $\begin{array}{c}40 \\
5\end{array}$ & ABSENCE DE TALENTS & 3 \\
\hline - érudit (dānišmand) & 1 & & \\
\hline - savoir religieux & 1 & & \\
\hline $\begin{array}{l}\text { - talents du lettré } \\
\text { (écriture, poésie, échecs) }\end{array}$ & 27 & - pas de talents (hunar na dāšht) & 3 \\
\hline $\begin{array}{l}\text { - talents du nomade } \\
\text { (chasse, tir à l'arc...) }\end{array}$ & 6 & & \\
\hline $\begin{array}{ll} & \text { SAGESSE } \\
\text { - sage ('āqil) } & \end{array}$ & $\begin{array}{c}12 \\
2\end{array}$ & $\begin{array}{l}\text { ABSENCE DE SAGESSE } \\
- \text { fou }(d \bar{\imath} v \bar{a} n a)\end{array}$ & $\begin{array}{c}12 \\
2\end{array}$ \\
\hline - humble ('alīm) & 4 & - orgueilleux (mutakkabir) & 3 \\
\hline - patience (sabr) & 4 & - pas de mesure (bī-tandiri $\overline{)}$ & 2 \\
\hline 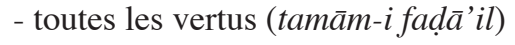 & 1 & & \\
\hline - parfait (kāmil) & 1 & - cruauté (avide de sang, tueur...) & 5 \\
\hline GÉNÉROSITÉ & 6 & AVARICE & 2 \\
\hline - généreux (sah̄̄, karīm) & 6 & - avare (mumsik, saht gīr) & 2 \\
\hline COURAGE & 16 & & \\
\hline $\begin{array}{l}\text { - brave, courageux (dilīr } r \text {, dilāavar, } \\
\text { šjuja' }{ }^{\prime}, \text { bahādur) }\end{array}$ & 16 & & \\
\hline DIGNITÉ DE SOUVERAIN & 14 & ABSENCE DE DIGNITÉ & 7 \\
\hline - conduite des qan (sîrat-i hāan) & 6 & $\begin{array}{l}\text { - vagabondage, misère } \\
(\bar{a} v \bar{a} \text { gard } \bar{\imath}, \text { falākat })\end{array}$ & 4 \\
\hline - principes royaux (ahkām-i mulkī) & 1 & - manque d'autorité (bī ihtiyārr) & 2 \\
\hline -attentif (mufattiš) & 7 & - négligent ( $\dot{g} \bar{a} f i l)$ & 1 \\
\hline BONNE CONDUITE & 8 & MAUVAISE CONDUITE & 16 \\
\hline - ascèse, dévotion (zāhid, ‘ābid) & 3 & - vie dissolue ('ayš kardan) & 3 \\
\hline - vie des saints, des prophètes... & 4 & - mensonge & 1 \\
\hline $\begin{array}{l}\text { - respect de la pratique musulmane } \\
\text { (jeûne) }\end{array}$ & 1 & $\begin{array}{l}\text { - non-respect de la pratique } \\
\text { (pas d'ablutions) }\end{array}$ & 1 \\
\hline & & - s'adonner à la boisson & 10 \\
\hline & & - aimer dormir & 1 \\
\hline Total des traits positifs & 110 & Total des traits négatifs & 43 \\
\hline
\end{tabular}

Fig. 13 : Mu'īn al-Dīn al-Națanzī, Muntahab al-tawārīh .

Tableau récapitulatif des appréciations de Mu'īn al-Dīn al-Națanzī sur les lignées turco-mongoles. 
Annexe XIII
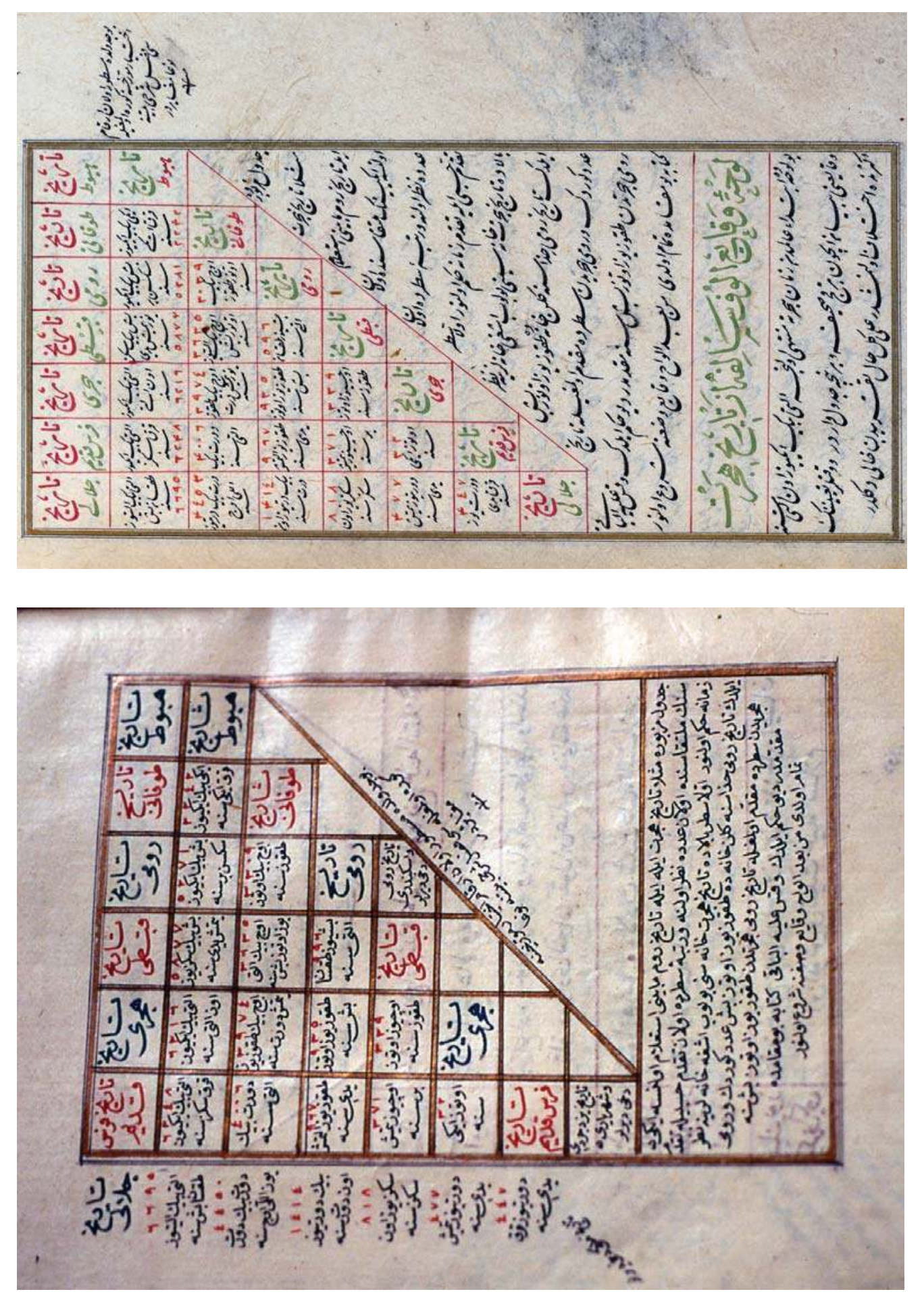

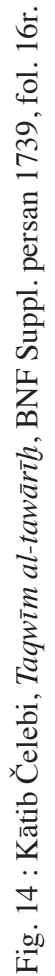


Annexe XIV
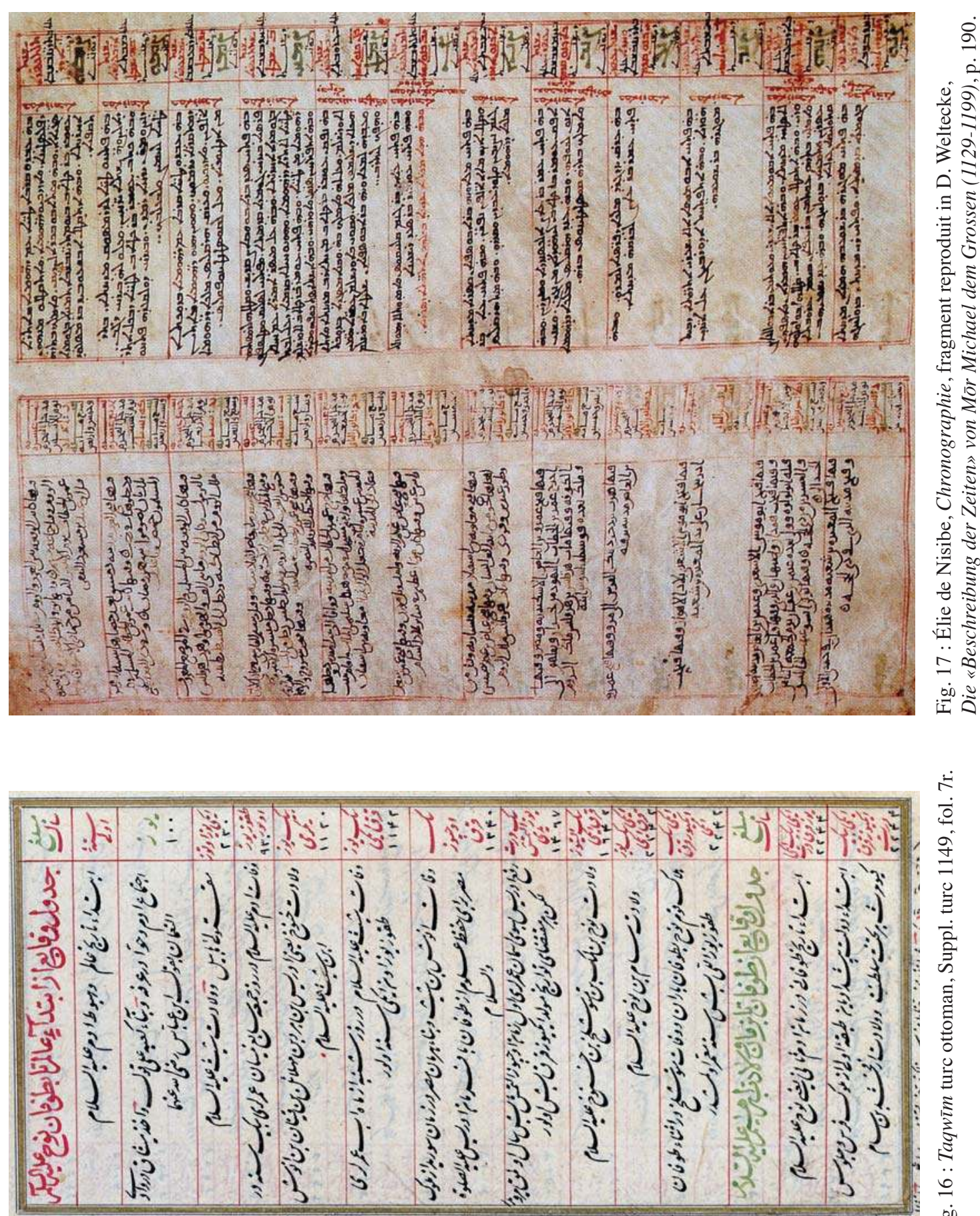

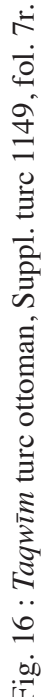

